\title{
El Metalenguaje XML y el Esquema de Tipo de Elemento
}

\author{
JUAN VOUTSSÁS MÁRQUES \\ CentroUniversitariodeInvestigacionesBibliotecológicas \\ de la UNAM, 04510, MéxicoD.F., Tel:56-23-03-29 \\ E-Mail: voutssás@servidor.unam.mx
}

\begin{abstract}
RESUMEN
En las últimas décadas se ha ido desarrollando una herramienta para el procesamiento de información electrónica conocida como los metalenguajes de marcado. Desde el formato "MARC" de los sesentas hasta el "XML" es ta ble ci do en nues tros días ha ha bi do sin duda una evolu ción en este sen tidobas tan te no table. Elpresentedocumen to tra ta dees tablecer una evolución histórica y funcional sobre los lenguajes de marcado, sus características, ventajas, limitaciones y sus expectativas.

Dentro de las especificaciones "XML" se ha desarrollado recientemente una técnica particular de declaración y manejo de la información documental conocida como "esquema de tipo de elemento" o simplemente es que mas, la cual va más allá de la sim ple y ya co no ci da de fi ni ción del do cu men to a tra vés de un des crip tor de tipo de do cu men to (DTD). Los esque mas nos ofre cen una po si bi li dad mu cho más am plia para de fi nir cla ses de documentos. Este trabajo intenta establecer esas posibilidades para po der en ten derla po ten cia li dad de los es que mas, su es ta do delarte, sus ten den cias y finalmen telaim portan cia que elde sa rrollo de es que mas es pecia les para cier tas cla ses de do cu men tos pue de te ner en nues tro medio bibliotecariomexicanoparaunmejores tablecimien toy explotación de las colecciones digitales.

Pa la bras Cla ve: Me ta len gua jes, Len gua jes de mar ca do do cu men tal, XML, HTML, SGML, MARC, DTD, Es que ma de tipo de ele men to, Es que mas, Bi blio te cas di gi ta les, De sa rrollo decolec ciones digitales, Tec nologías de in for mación
\end{abstract}

\section{METALANGUAGE XML AND THE TYPE \\ OF ELEMENT SCHEME \\ JUAN VOUTSSÁS-MÁRQUES}

\begin{abstract}
During the last dec ades a tool for elec tronic proc ess ing known as markup lan guages has been and is still be ing de veloped. From the MARC Format of the 60's to the XML es tab lished in our days, a very clear evo lu tion has, no doubt, taken place. This paper intends to settle the issue of the his tori cal and func tional evolu tion of the mark- up lan guages and of their characteristics, advantages, limitations and expectations.
\end{abstract}

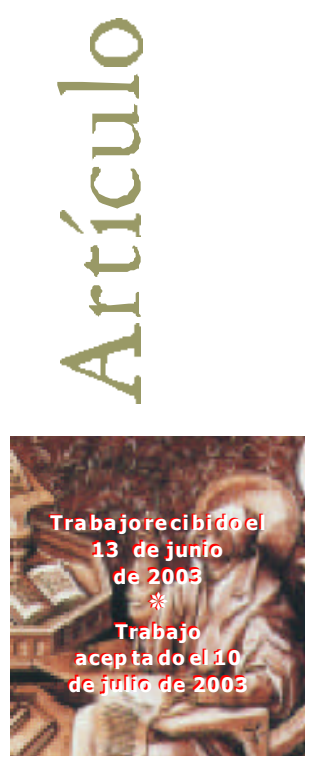


Within the specifications of XML a particular technique for the declaration and handling of the documentary information has been developed recently. The te chnique is known as Type of Ele mentSche me, or sim ply Sche mes, but it goes well beyond the sim ple and very well known de fi ni tion of do cu ment through a Descriptor of Type of Document (DTD). The schemes offer a much wider possibility for defining classes of documents. This paper attempts to establish the se pos sibilitiesin ordertounders tand the po ten tiality of Sche mes, its sta te of art, its ten den cies and also the im por tan ce that the development of special schemes may have for certain classes of documents in our Mexican library environment for a better implementation and exploitation of digital collections.

Key Words: Metalanguages; Document Markup Languages; XML; HTML; SGML; MARC; DTD; Esquemas; Digital Library; Digital Collection Development; Information Technology

\section{ANTECEDENTES}

D es dela déca da de los se sen tas, el perso nal téc nico rela cio na do con elin tercambio de documentos por medio de una computadora comenzó a preocuparse por estructurar esa información en una forma normalizada que facilitara el intercambio y la manipulación de esos materiales. En el ámbito de las bibliotecas surgió el proyecto del formato "MARC", Macbine Readable Cataloguing), pionero en esta temática.Durante 1965laBibliotecadelCongresodelosEsta dos unidos(LC) es tableció un proyecto piloto denominado "MARC I" cuyo objetivo era definir una metodología para crear registros catalográficos en un formato legible por una computadora. Un pro yec to si milar apa re ció en el Reino Uni do en esa mis ma épo ca ordenado por el Con se jo de la Biblio te ca Bri tánica (Bri tish Li brary) con el fin deesta ble cerun for ma to para re gis trar y ex plo tarlos re gis tros que se rían usa dos en la $\mathrm{Bi}$ blio gra fía Británica. A esteproyec to selella mó “BNB MARC” (BritishNationalBibliography with MAcbine Readable Cataloguing). Ambos proyectos derivaron en poco tiempo en una cooperaciónen el ambiente de las bibliotecasanglo-sajonasydieron origen, en 1968, al proyecto "MARC II". El resultado fue el formato ampliamente conocido para intercambiar regis troscatalográ ficosdemonogra fías vía electrónica, el cual fue complementado poco después con los consecuentes formatos para publicacionesperiódicas, mapas, discos, etcétera, has ta comple tarprácticamentetodo los tipos de documentos que existen en las bibliotecas.

Con el tiem po MARC fue tan exi to soy tan am plia men te utiliza do en todo el mundo que empezaron ausarse múl tiples va rian tes del mis mo, has talle gara unas vein te que fueron adaptadas a las nuevas necesidades deotras co munidades enel pla neta. Para 1977 se definió el formatouniversal "UNIMARC" como una es pe cie de "es peran to" paraMARCconobje to de quelos dis tin tos "MARCs" pudieranin tercambiarre gistrosentresíusando "UNIMARC"comointermediario.Además el forma totambién 
empezóa serde finidoparaotras en tidades de da tos más alládela sim ple pro ducción de ca tálogos, comolos re gis tros de au to ridad, pues su di seño permitía que fue rausa do en prác tica men te cual quier pla ta forma de cóm pu to del mundo. Debido asu ampliaaceptación MARC se con vir tió en nor maANSI para la unión americana en 1971 (Z39.2 - 1971) y en norma ISO internacional en 1973 (ISO 2709: 1973 E). Está de más ahondar sobre lo que MARC representó paraelregis troelectrónicodeinformación. [LibraryofCongress, 2003]

En esencia, MARC no ha sido nunca un catálogo ni un método para catalogar, como mu chos han pen sa do a lo lar go del tiem po. La gran apor ta ción deMARC al manejo documental, y que ha sido seguida por muchos formatos posteriormente has ta nues tros días, fue la de aso ciar una "eti que ta" a cada uno de los ele men tos que conforman una ficha catalográfica, con objeto de que las computadoras puedan identificar y catalogar sus partes y posteriormente efectuar múltiples acciones con ellas en beneficio de las personas. Este principio tan simple y tan obvio en nuestra épocamarcóun hito enlahis to ria delmanejo documental pormediode computadoras. Hoy sehaso fis tica do y diver sifica do enor memen te, peroel principiosemantienehastanuestros días. [Ageneralin troduction tomarc] [Iflanet. Whatismar]

Dehe cho, este concep to de aso ciarcada ele men to de una ficha, y pos te rior mente de cualquierotro docu men to, con una "etique ta" (lla ma da en in glés la bel, tag o token) fue conociéndose como "marcado" del documento, y ha sido de tal trascendencia que to dos los forma tos que han apa re cido pos te rior men te para el re gis tro de do cur men tos se ba sanen el con cep to del marcado, y asílo os ten tan en su nombre (markupo mark up.) Abun da rémás adelan te en este con cep to.Porlo an te rior, MARC pue de ser consideradoel "abuelo" detodosloslenguajesdemarcadodocumentalparacompu tado raque existenhoy.

Fuera de las bibliotecas, a nivel general y por esa mis ma épo ca de fi nes de los sesentas, la empresa IBM creó "GML" (Generalized Markup Language) o "Lenguaje de marcado generalizado"para contender con las necesidadesde sus propios sistemas internos depublica ción. Ellosusa ron GMLparapro du cirde mane ra normalizada, li bros, re portes, ma nua les y otros tipos de do cu men tos a par tirde unúnico conjun to de archivos originales en una computadora. Muchas otras soluciones fueron introducidas por otras organizaciones para estructurar información documental, pero nadarealmentequeabarcaraestepropósitoagranescala.

Laprimeratecnologíadeestructuracióndeinformacióndocumentalnormalizada de cierta signi fi can cia fue el "SGML" (StandardGeneralized Markup Language) o "Lenguaje de marcado generalizado estándar", en lo sucesivo "SGML", el cual también provinodelaempresa IBMa prin cipios delos ochen tas. Estelen gua je fue crea docon el fin de formatear y organizar la documentación legal dentro de esa empresa, pero posteriormentefue ex pan dido yadap ta do para ser usa do en una am plia va rie dad de empresas como un estándar para manejar todo tipo de información y para 1986 se convirtióen una normaISO [ISO 8879,1986]. 
Si bien SGMLes extremadamentepoderoso, esigualmentecomplejoyrequiere de una considerable cantidad de programación adicional para procesarlo. Debido a su compleji dad ylos re cursos ex traque de manda, SGML no era una op ción via ble para representarhipertex tosenlasprimerasépocasdelaInternet, dadaslasdimensiones y limitaciones delosequiposdeesaépoca.

En 1989, Tim Berners-Leey Anders Berglund, dosinves tigado resdelLa bora torio Europeo de Partículas Físicas (CERN), crearon un lenguaje basado en etiquetas paramarcardocu men tos técni cos yserés tos compartidosenInternet.Estelenguaje fue expandido en 1990 a una versión simplificadadel SGMLlla mada HTML (HyperText Markup Language) "Lenguaje de marcado de hipertextos", y ha llegado a ser el formatoestándarparaelmanejodein formación enla " $W e b$ ”.

HTML podía representarperfectamenteinformación estática en una página Web; es decir, textos previamente establecidos, imágenes, botones, ligas, etcétera, pero pronto hubo necesidad de ligar las páginas a bases de datos con el fin de traer a las pantallascatálogos, es ta dosde cuenta, tex tos completos, etcétera; esdecir,información dinámica o cam bian te, para lo cualHTMLno fuediseñado. Algunosaña di dos han surgidopara HTMLen los úl timos años, para re sol veresta proble mática, al gu nos de ellos muy exi to sos, como los deriva dos del len gua je "Java", y otros han caído ya en el olvido. Al final, HTML comienza a estar dema siado "remenda do" y re sul taya complejoparapodersatisfacerlos requerimien tos del manejodein formacióndelaactualidad.[Teasdale,1995]

Comore sul ta do de esto mu chos téc ni cos han em peza do a volversus ojos nuevamen te ha ciaSGML, ya que su compleji dad era de origen y no re sul ta dode adiciones, y siemprehatenidocapacidadparamanejardocumen toscomplejos devariadostipos. Otrosgrupos depersonasempe za ronarees cribirunaversión simplificadade SGML pero ca paz de con ten der con las ca ren cias de HTML; de ahísurge un nue volen gua je llamado XML: el (eXtensible Markup Language) o "Lenguaje de marcado extensible", en lo sucesivo "XML”. En 1996, el "Consorcio para el desarrollo de la World Wide Web", conocidocomo elW3C, sentólas bases paraes tos de sa rrollos. Se es tablecieron las ven ta jas pro pias delSGML: es tructura, ex ten sibilidadyvalida ción, yse creó ungru pode trabajoqueestablecieralas bases paraun nuevolenguajedemarcadoqueconser va ra las ven tajas cen tra les delSGMLy que tu vie ra la sim plicidad delHTML; esto es, un SGML “ae ro di ná mico" parala Web. El re sul ta do fue que en 1998 se pu bli ca ronlas es pecificaciones dela primeraversión delXML[Ex tensible MarkupLanguage1.0,2000]. En cuantoa dimensiones, laespecifica ción XML re sul tó ser de me nos de una déci ma par te de la deSGML, conlocual pue de es ti marseelgra do de compac ta ción. [MSDN on Line, 2000]. Des de ese año a la fe cha, el con sor cio W3C se ha cons ti tui do comolains tanciaoficialanivelmundialparadefiniciones delestándarXML. 


\section{CONCEPTOSBÁSICOS}

\section{Metalenguaje}

Si se observa la literatura al respecto, puede notarse que HTML, SGML, XML y otros entes del manejo de información documental son denominados como "lenguajes", pero más propiamente dicho, se trata de "metalenguajes". ¿Qué significa realmen te estetérmino? esimpor tante acla rarlo parapodercomprenderel con tex to deesasherramientas.

Elcon ceptode "me talenguaje" no es sim pley preten de es table cerlas reglas porlas cua les un len gua je dado pue de serde finido o exa mi na do formalmente, pero existen muchasclases delenguajes:loslenguajes quelos humanosusa mos paracomunicarnos cotidianamente,español, inglés, francés, etcéte ra;lenguajes paraprogramarunacomputadora,Fortran,Algol,“C”,Pascal,Cobol,Java,etcétera;elálgebra,etcétera.

Así pues exis ten mu chos me ta len guajes; más delos len gua jes que pue den ser crea dos. Inclusounlenguaje cualquiera que habla moslaspersonasincluyevarios "sublenguajes" o "argots" pro pios de una pro fe sión ocla se so cial. Dis tin gui mos por ejem plo ellen guajepropio delos médicos den trode un len guaje dado, o el del perso nal téc nico de cómputo, etcétera. Los enfoques pueden ser entonces filológicos, matemáticos, computacionales, etcétera. Para fines de este tra ba jo nos cen tra re mos enlos len guajes "documentales",quesonlos denuestrointerés.

Deestaformapodemosestablecerunmetalenguajeparadefinirunlenguajedado y por tan to, definiremosentoncesdeunaformasimple"metalenguaje"como un medio para definir formalmente un lenguaje dado. Si bien entran también aquíconsideraciones de sintaxis, contex to, objetos, etcétera, concentrándonosen el tema que nos ocu pa; es de cirlos do cu men tos, re sul ta quelos me talen guajes (MARC, SGML, HTML, XML) son un me dio para des cri bir un len guaje de mar ca do; di cho de otra forma, sonmetalen guajes para de finirformalmen teunlen guajedemarcadode documentos.

Las reglas para de fi nirel marca do de do cu men tos, su sin taxis, suslimita cio nes y carac te rís ticas es tán den trode cadaunode esos en tes (MARC, SGML, HTML, XML); esdecir, ellosinterna mentevan con forman do todo un len gua jepara marcarocodifi cardocumen tos. Ellenguajeestáporlotan toin trínsecamentecontenidoencadauno de ellos, pero la manera de presentar el conjunto de las reglas y definiciones de ese lenguaje es un metalenguaje. Por eso decimos que estas herramientas son metalenguajesmáspropiamentedichoquelenguajes.

Históricamente, la palabra marcado (markup) vie ne delas ins truc cio nes que se le da bana un im pre soro tipó gra fo so bre cómo de bería im primirse un cier to pa saje de un tex to: las negritas, los sub ra ya dos, la ti pogra fía, el tipo yel ta mañodele tra, los símbolos especiales, etcétera, se señalaban con anotaciones o "marcas" al margen del tex to; de ahíel nombre. Con formelos tex tos se fue ronau to ma tizan do, el término se fue extendiendo hasta cubrir toda clase de códigos de marcado insertados en esos 
textos electrónicos con objeto de incrustar una serie de características propias de cadasecciónopartedeltex to.

De este con cep to se des pren de que el marca do es una co di fi ca ción del tex to, gracias a la cual cada por ción del mis mo seva hacien do ex plíci ta y toma forma en un contexto. De hecho, y hablando formalmente, todos los textos han tenido desde hace largo tiem po una ma ne ra de ha cer se ex plícitos y en ten dibles allec tor. Los sig nos de pun tua ción, los acen tos, los es pa cios en tre pa la bras, el uso de ma yús culas, los pá rrafos, los ca pítulos, los in cisos, etcé tera, sehanagrega doalos tex tos des de hace mu cho tiem po para que sus lec to res pue dan leer ex plíci ta men te un conjun to de le tras y darles un sen tido. Ima gí ne se ellec tor un tex to en el cual to das las le tras es tán con tiguas, sin espacios ni puntuaciones ni nada adicional, y podrá darse cuenta de la enorme ayuda que estos elementos proporcionan para hacer explícitas estas palabras. Si el lectornoseloimaginaanaliceentoncesestetex to:

adanyrazaazarynada

Como puede observarse, el texto anterior difícilmente puede ser interpretado al primer golpe de vista; es necesario leerlo y releerlo varias veces para que el cerebro humano, tan avezadoen este tipo de ac tivida des, pue da es ta ble cerpa tro nes, cortes, analogías, ydescifrarsusignificadoysucontex to. Puestodeestaforma:

\section{palíndromo:}

\section{Adán y raza, azar y nada}

es mu cho más fá cil dein terpre tar. Cier tas con ven ciones de es pa cios y pun tua ciónlo hacen explícito. Incluso, gracias al encabezado los lectores se cercioraron de que el tex to es un pa lín dro mo, es de cir, algo que pue de ser leídoigual men te deiz quier da a dere chaquedede rechaaiz quierda. Algunos no se ha brán perca ta do de ello has ta que elencabezado,esdecir,cierto marcado, lohizoexplícito.

Del mismo modo, y en ello radica su importancia, un lenguaje de marcado debe permitirhacerexplícitoun tex to para los sistemas y las máquinas que los ope ran electrónicamente: además de dónde empieza y termina cada palabra, lo cual puede lo grar se con es pa cios, nos in di ca dón de em pie za una par te de un tex to en un do cumen to y dón de aca ba, qué par tes tie ne ese do cu men to y cómo sella man, et cé te ra. El lenguaje de marca does en tonces un conjun to deconvencionesprees ta bleci das que seusan demaneraconjun tay ordena da para marcaro codi ficarun tex tode modoque éstepue da seren ten di do ex plí ci ta men te por má qui nas para que és tas, a su vez, selo puedanhacerexplícitoalaspersonas.

Unlenguajedemarcadodebees pecificaren toncescuálesmarcas sonpermitidas, cuáles sonobliga to riasu op cionales, cómodeben dife ren ciarseesas marcas del tex to propiamentedichoy,porsupuesto, quésignificacadamarca.

Tratarédeilus trarconunejemplolostemas anteriormenteexpuestos. 
Usted quiere describir una parte de un documento que es la dirección postal de una per so na dada, la cual, como sa be mos, está for ma da a su vez por va rios ele mentos. ¿Cómodescribiríamosesta entidadenlenguajellanoperoalavezformalmente?

Talvezse ría algocomoesto:

Una dirección postal consiste de: una parte-que-tiene-el-nombre,seguida de una parte-que-tiene-la-dirección, seguida de una parte-que-tiene-el-código-postal. Laparte-que-tiene-el-nombre consiste de: un nombre-de-pila, seguido de un apellido-paterno, seguido de un apellido-materno, si se tiene. Laparte-que-tiene-la-dirección con sis te de: un nom bre-de-calle, se gui do de un número-exterior, seguido (silohubie se) de un núme ro-inte rior, se gui do de un nombre-de-colonia.

Laparte-que-tiene-el-código-postalconsiste de: un nombre-de-ciudad, seguido de un nombre-de-entidad-federativa, seguido deun código-postal Un código-postal consiste de un número de cinco dígitos.

Usemosunmetalenguaje parades cribiresto. Porsimplicidad, usaremosla"forma de Backus- Naur" (Backus- Naur Form o BNF), un metalenguaje muy utilizadopara de finirlengua jes de progra ma ción des de 1959 yque toma el nombre de sus creado res John Backusy PeterNaur. [FreeDictionary....2000].

Lasbasesdeconstruccióndelmetalenguajesonlassiguientes;primerolossímbolos:

::=: $\quad$ significa "se define como"

|: $\quad$ significa "O"

$<>$ : $\quad$ corchetes angulares usados para encerrar nombres de categorías o "entes".

\{\}$: \quad$ corchetes de llave usados para encerrar nombres de categorías opcionales.

“ ": Texto entre comillas que se integra literalmente tal como está escrito.

$<$ EOL $>$ : fin de la línea de definición

La manera de usar la forma de Backus-Naur es la siguiente: se pone del lado izquierdolaen tidad a de finiry dellado de recholadefinición, separadas por el signo de "se de finecomo". Se trata de pasardelogene rala lo particulary se evi taráa todacosta la tau to lo gía; es de cirquela parte a de fi nirseaigual quela de finida. No obs tan te, una parte de lo que está a la derecha como definida pue de ser par te de lo que se de fi ne. Porejem plo, en elálge bra, la ex pre sión $n=n+1$ no pue de exis tir, ya que de acuer do a sus reglas, no hay nin gún nú me ro que sea igual a él mis mo más uno. En no ta ción $B N F$ $n::=n+1$ sípue de exis tiry signi fica quen se de fine comoél mis mo in cre men ta do en una uni dad. Perfec ta ma ne ra de es ta ble cer un con ta dor. Sise ob serva el ejem plo anterior, se ve que $n$ forma parte delo que se de fine y delo de finido, pero es co rrec to bajoestas reglasynoesunatautología. 
Sien al gún mo men to una de fini ción pue de ser de finida a su vezen componen tes mássimples,esanuevadefiniciónseescribeacontinuación.

De acuerdo con lo anterior, podemos definir más formalmente una dirección postalsegúnlanotacióndeBackus-Naurcomo:

$<$ dirección-postal $>:$ := <parte-que-tiene-el-nombre $><$ parte-que-tiene-la-direc

ción> <parte-que-tiene-el-código-postal $>$

$<$ parte-que-tiene-el-nombre $>::=<$ nombre-de-pila $><$ apellido-paterno $>$ \{apellidomaterno\}

$<$ nom bre-de-pila $>: .=<$ nom bre $>\mid<$ ini cial $>$ "." $<$ EOL $>$

$\mid<$ nombre $><$ nombre-de-pila $>$

$<$ parte-que-tiene-la-dirección $>::=<$ nombre-de-calle $><$ número-exterior $>$ \{número-interior\}<nombre-de-colonia>

$<$ parte-que-tiene-el-código-postal $>::=<$ nombre-de-ciudad $><$ nombre-de-entidad-

federativa $><$ código-postal $>$

$<$ código-postal $::=<$ dígito > < dígito><dígito><dígito><dígito>

$<$ dí gi to $::=0|1| 2|3| 4|5| 6|7| 8 \mid 9$

De acuer do con este ejem plo, y utilizan do la no ta ciónBNFpodemosob servarlo siguiente:

* Hemos de finidodirec ción-pos tal comounaca te go ría for mada por tres partes (nombre, direc ción y có digo) yés tas de ben exis tirsiem prey en eseorden.

* Hemos definido a su vez al nombre como una categoría formada por el nombre-de-pila, seguido por el apellido paterno y opcionalmente, el apellido materno, y en ese orden.

* Hemos definido también al nombre de pila como una categoría que puede es tar forma da por dos po si bili da des: una de ellas es un nom bre o una ini cial más un punto obligatorio; la otra posibilidad es compuesta, y puede estar formada por un nombre seguido de lo que habíamos ya utilizado como nombre de pila; es decir, el concepto se vuelve recursivo. Esto es, puedo usar un nom bre más otro nom bre, o un nom bre más una ini cial, o una ini cial más un nombre, et céte ra. Di cho de ma ne ra prác tica, un nom bre de pila puede ser José o J. o JoséL Luis o J. Luis o José L. o J. L. . Al usar el conceptode recursividad una en tidad se de fine como un nom bre se guido de lo que se había utilizado como nombre con anterioridad; es decir, se pueden ir acumulan do. To dos los nom bres men cio na dos en el ejem plo ca ben den tro deesta de fini ción. Elsímbolo $<$ EOL $>$ in di ca que lo del primerren glón es apar te de lo del segundo y no deben mezclarse.

* Hemos definido también que la dirección está formada por el nombre de unacalle, segui do de un nú me roex te riory, opcio nalmen te, un nú mero in terior, seguido de un nombre de colonia, en ese orden.

* Hemos definido que el código postal está formado por el nombre de una ciudad, seguido por el nom bre de una en tidad fe de ra ti va y el nú me ro de un código postal, en ese orden. 
Hemos definido que un código postal está formado por cinco dígitos consecutivos.

* Hemos de finidoa un dígi to como cual quie ra de los gua ris mos del ceroal9.

Sibien no he mos de finido una se rie devalida cio nes yres tric ciones con res pecto a estos po si bles da tos, ya que se tra ta de un ejem plo muy sen cillo como pue de ob servarse, la de fíni ción de un entella madodi rec ción pos talesposible, precisaypocoambiguacuandousa mosunme talenguaje; en este casola no ta ción BNF. Esto es más fácil que tratar de explicarlo en lenguaje corriente, ya que habría que estar haciendo muchas explicaciones so bre cada ele men to, yade más el re sul ta do es una de fi ni ción quetienemayorformalidad.Ademásestas definicionespuedenserinterpretadaspor unprogramadecom pu ta dor. Puedeha cer se en ton ces que ese pro gra ma es cribalas ca te gorías en si tios pre ci sos den tro deuna pantalla, hoja oetiqueta de pa pel:primero el nom bre, lue go la di rec ción y lue go el có digo. Pue de ha cer se un lis ta do sólo delos nombres delas personas yomitirselode más; puedehacerse un lis tadoqueordenelos nombresalfabéticamenteporapellidos, etcétera.Todas estasaccionesseríanmuydifíciles de efec tuar si el nom brey la di rec ción de una per so na fue sen una sola en ti dad es crita de co rrido, con locualuna má qui na severía im po sibili ta da de dis tinguircada unade esaspartes.

Cabe ha cer no tar que esta no ta ción de Backus- Naur es sólo un ejem plo de un metalen guaje sen cillo parailus trar con cep tos bá si cos so bre este tó pico. No seusa en la prác tica para des cribirtex tos ya que ca rece de unbuen núme ro de elemen tos ne ce sa rios para realizar un correcto encodificadode ellos mis mos. Para ello se han crea do metalenguajespropiosparaeseefecto.

\section{HTML (HYPER-TEXT MARKUP LANGUAGE)}

Como ya se ha men cio na do, a prin ci pios de los no ven tas se creó el World Wide Web, $W W W$, o simplementeWeb, comounmecanismopara editaryaccederain formacióna nivelmundialconbaseenlas tecnologías deIn ternetored globaldecomputadoras. En uniniciolain forma ción que viaja ba en el in ternetera sólo de tipo tex to, y las ins trucciones de bían ser te clea das a modo de co mandos. Elad ve ni mien to dela Web le agre ga interfa ses gráficas ala dis tribu cióndein forma ción; es de cirqueunapá ginadela web contiene ahora además de textos, imágenes (fotografías, dibujos, diagramas, etcétera), así como otros ele men tos de ac ce so más có mo dos para el usua rio: bo to nes para se lec cionaropciones, menús, etcétera. Agrega tambiénunele men to muy importante: el hipervínculo; es decir una "liga” con otro texto, que puede estar en esa misma página o en cualquierotra, inclu so en otra computa dora, sis tema o has ta otropaís. Gra ciasalhipervínculo, elusua rio pue de, al po sicio narse so bre un pun to enla panta lla, di ri gir sehacia otroconjun to deinforma ción desuin terés sobrelaWeb. Posteriormen te seagrega ron 
al me dio sonidos eimáge nes en mo vimien to, como anima cio nes o video, para ha cer delaediciónenlaWebunambienterealmentemultimedios.

Precisamente para poder efectuar esta edición de información por medio de la Websedesa rrolla elmetalenguaje HTML que como ya se ha vis to nace como un subconjun to oaplica ción muyes pecífica deSGML, del cual sólo se de sea ban al gu nas de suscaracterísticasque permitierandescribirlaspartes deunapáginapensandosobre todo en cómoiban a verse so brela pan talla.Lo prin cipal era la dis tribu ción física de la in formación so brelapantalla,esdecirel lay out:dón dedeben quedarlosencabezados ole tre ros, su tipo dele tra, ta maño y colo res; el di seño y co lo res delos fon dos; ubicación, color,tamaño, etcétera depárra fos sobrela pan talla;laubica ción, tamaño,etcétera, de lasimágenes so brelapan talla; los bo to nesparaseleccionaralgunaopción d e entreunmenú;lasligasconotraspáginas, etcétera.

Como pue de verse HTML po día de finir un cier to nú me ro deen tida des en una pá gina a la cualpo día arribarunusua rio, ybrin darle accesoa lain formación. Peroadolece de un se rio de fec to: los tex tos en suin te rior son ma ne ja dos sola men te como eso, como "tex tos"; es de cir, como un con jun to de pa la bras con tiguas, sin nin gún dis cernimientodesucontex to, susignificado, suponderación, sus relaciones, etcétera. Por esta ra zón al mul tiplicar se el nú me ro de pá ginas en la red sehizo nece sa rio un meca nismo que nos permitiera saber en dónde podría aparecer algo sobre un tópico de nues tro in te rés. Na cen asílos "bus ca do res", con el fin de po der bus car y en con trar algo en la red. El pro ble ma es que los tex tos sólo es tán mar ca dos como tex tos, ca denasde palabrassinningu napondera ción so bre sucontex to o suspartes.Las primeras búsque das sólo ha cen un barridobasán dose en la "fuerzabru ta" de la com pu tado ra que tra ta deiden ti fi car las pala bras con te nidas den tro de esos tex tos. To dos los que hemos navega doyutiliza do lared sa be mos el resul ta dode es tos procesos:docenas, cientos yhas tamiles derefe ren ciasinútiles llenas de "ruido" eirrelevan tes paranuestropropósito.Quienes conocenalgo deca taloga ción bibliográ fica sabenqueesmuy diferentebuscar por ejemplo, a Oc ta vio Paz como au tor, como tí tu lo o como tema en una obra. Peor aún si sólo buscamos por "paz" fuera de todo contexto, pues encontraremos a otras personas apellidadas o llamadas "Paz", pero también noticias sobre la paz, o la fal ta de ella, do cu men tos, tra tados, es tu dios, et céte ra, que nos ha blandeesteestado so cialyquenada tie nen quevercon nues tro Premio Nobel.

Peoraún fue cuan dolaspági nas comenza ron aserpuerta de en trada paraenormes repositorios de información, digamos un catálogodebiblio teca¿cómoen contrarla in for ma ción que está den tro de esa base de da tos, pero que fí si ca men te no está en la página Web? Las pala bras para re cupe rar docu men tos usa das como lla veses tánasociadas a la pá gi na en sí mis ma, no a los ban cos de da tos liga dos a ellas. Esa in for maciónestáperdidaparalarecuperación.

Losbuscado res tuvieronqueirseso fis tican do parapodercon ten derconesteproblema y hoy varios me ca nis mos se han in tro du cido con este fin, comolos "meta datos",los "bus cadores in teligentes"y al gunosotros adita men toscomo:fre cuencia de la palabraenunapágina, proximidadocon tigüidadentre ellas, etcétera.Bas tantese 
ha avan za do en este sen tido, y de be mos re co no cer que en la ac tua lidad hay pá gi nas conmejoresín dices ybusca doresreal mentemás ade cua dospara en con trarin forma ción pertinente. Pero el pro ble ma vie ne de origen, des de la ma ne ra en que la in forma ción es prepara day des crita en HTML. Este for ma to tiene al gu nas otras de ficiencias, entrelascualeslas más rele vantessonque HTML:

No es extensible; es de cir, el conjun to de etique tas es ce rra do y na diepue de definir sus propias etiquetas para requerimientos específicos. Por ejemplo, en el mundo bibliográfico querríamos tener etiquetas tales como <autor $>$, $<$ título $>\mathrm{o}<\mathrm{ISBN}>$ en lugar de la etiqueta genérica $<\mathrm{p}>$ de párrafo.

No permite representar las especificaciones de las estructuras de los datos, como se requiere en la creación y uso de bases de datos.

* No provee soporte para validar los datos.

HTML, por lo tan to, no manejalos as pec tos de con teni do y no pue de ser uti lizado,porejemplo, paracalificarelcontenidodepáginas Web. HTML sólo se en fo ca a la pre sen ta ción delain for ma ción. Más allá de esto cual quier cosa re quie re de un conside rable es fuerzo de pro gra ma ción adi cio nal, ya sea en forma de applets o de aplicacionesodeprogramas.

No obstante lo an te rior, se ría un gra ve errormenos pre ciarel HTML. Este es tándar ha logrado resultados inusitados en la expansión de la divulgación documental electrónica en los últimos años. Gracias a él la Web ha llegado a ser lo que es hoy en día. HTMLreforzó elhechodequela plataformadecómpu to fue seirrelevanteparael in tercam bio dein for ma ción, y creó las ba ses del me ca nis mo de trans por te para mover documentos a lo largo de distintas redes (lo que llegó a ser el protocolo HTTP, Hyper-Text Transport Protocole), y también el esquema de direccionamiento de documentos tanto locales como remotos (lo que llegaría a ser el direccionamiento URL UniversalResource Locator). Bas tan te se hizo conHTML a pe sar de su es bel tez y sus limitaciones, simplemente resulta que las necesidades de manejo documental siguen cre cien do y em pie za a ver se li mi ta do para con ten dercon ellas. Como mu chas otras gran des he rra mien tas el HTMLestá siendo rebasa do por el mis modina mis mo de la in for ma ción de la red. El con sor cioW3Chacon sideradootras opcionesquepue dan contender con esas de man das y es por ello queSGML es vuel to a to mar en cuen ta y nacen nuevos for ma tos comoXML o XHTML. Sin em bargo por un buen tiem po seguramenteseseguiránviendomu chasaplica cionesdesarrolladasen HTML. La apa rición de XML y su uso seguramente se implantarán por me dio de un cam bio gra dual delosdesarrollosactualesynomedianteunasustitucióninmediata.

Si bien los esfuerzos del grupo de trabajo del W3C se concentraron durante un tiempoenlade finición del XML, comolohemos yamencionado, re cientemente seli beraronlasespecificacionesdeunaprimeraversióndeunmetalenguajedenominado XHTML(eXten sibleHyper-TextMarkupLanguage) compatible conXML, cu yas especificacioneshansidoliberadasconanterioridad por el mismoconsorcio[XHTML 
1.0]. En lo per so nal no me que da muy cla ro por qué exis ten dos su ce so res de HTML provenientes de la misma fuente. Como ya se ha explicado, HTML se veía cercado poruna serie delimitacio nesque eran re suel tas conbase en aplica ciones adicio nales, y porelloungrupo de trabajo de sa rrolla un nuevo me talengua jeque, con tenien doal anterior (HTML), pueda con ten der con las ne ce si da des ac tua les, y por esto se de sarrollaylibe raasíla prime raversión de XML; has taahítodo sue na muylógico. Porqué el mis mo con sor cio li be ra des pués una nue va ver sión de TML y la llama XTML y es compa tiblecon MLno está cla ro y crea mu cho ma yor con fu sión en los de sa rro lla doresal no sa berés tos si sus nuevas aplica ciones provenien tesdeHMLdeberánhacerse en XHML o en ML. Pien so que elW3Cqui socondescendercondiversas fac cionesde gru pos de de sa rro lla do res y acep tó esta nue va ver sión delHTML convertida a XML con ob je to de darlegus to a to dos, perome pare ce quela versión con más fu tu ro seráa lalargaladenominadaXML.

\section{SGML (Standard Generalized Markup Language ISO 8879:1986)}

Como ya se ha comen ta do, elSGML sir vió de base al HTML. El primero, mucho más com ple to que su su ce sor, ade más delos as pec tos pro pios dela pre sen ta ción del documentopuedecontenerunaomásdefinicionesdetiposdedocumentos(DTD, o “DocumentTypeDefinition'), las cuales son des crip cio nes formales de la sin ta xis delos do cumen tos que se le asig nan a cada tipo de ellos que se de sea de finir. Por lo tan to, se requiere de una definición DTD para poder interpretar y verificar un documento SGML.

SGML tiene en tre sus prin cipa les ca rac te rís ticas ven tajo sas; prime ro, el serun están darno propie ta rio, es de cir, que no está ata do a una marca, pa ten te o com pa ñía en particular, yquees apo ya do porungran nú me ro de provee do res de software. Por ello, un do cu men to que cum pla con los es tán da res SGML tendrá una vida más larga que unoba sadoenunes tán darpropieta rio. Ensegundolugar, los documentosco difica dos bajoSGMLaún son bas tan telegibles paralas personas y portantoalavezlegibles para los programas de computadora. En tercer lugar, los documentos bajo SGML des criben la es truc tu ra de los da tos y su se mán ti ca, y no sólo la ma ne ra en que vana serpresentadosenlapantalla.

Entre sus desventajaspodemos men cio nar: pri me ro, el he cho de que es muygeneralizado, queincluyeespecificacionesparticularesparatodo tipodedocumentos y que se vuel ve muy com ple jo: sus es pe cifi ca cio nes se ex tien den por más de 500 pági nas. En segundo lugar, mu chas de esas es pe ci fica cio nes son irrele van tes para eluso del do cu men to en la Web, lo cual las hace su per fluas cuan do ése es el uso que se preten de darle al do cu men to. Dado que hay mu chas op cio nes den tro de éste, la in te roperabilidadentrediversasempresassereducesensiblemente.

Por lo anterior, los principales usos de SGML se han dado en ambientes "cerrados" don de es máso menos fácil con tro larla es tan da ri za ción de do cu mentos, tales 
comolossectores militares, dein teligencia, demanufacturadeaeronaves, empresas depublicación, grandessistemasdearchivo,etcétera.[SGMLISO8879,1986].

\section{XML (eXtensible Markup Language)}

Enlosanterio rescapítuloshemos vis toya quelas ven tajasydesven tajasde HTML y SGML se contraponen: HTML es práctico y esbelto, pero ha sido rebasado por las nece sidades del mo men to enla Web,y GML, sien do mu cho más com ple to tie ne muchos ele men tos im prác ti cos para su uso en la red. He mos vis to tam bién que elW3C, tratandodeconten derconesta problemática, favo re ció elde sa rrollodeun nuevoes tán darque tra ta se de to mar "lo mejor de am bos mun dos" yes asícomo nace el nuevo estándar XML, que pretende combinar las mayores capacidades descriptivas y semánticas del SGML con lo práctico, sencillo yuniversal delHTML. Podríaafirmarse que se tra ta de "laversióncorregiday aumenta dade HTML" o "un SGML ae rodinámicoparalaWeb".

En efec to,XML pre sen ta una se rie de ven tajas prác ti cas que lo ha cen ideal para ir sustituyendoal HTMLenlaweb, helas aquí:

* Sus especificaciones vienen contenidas en sólo 26 páginas.

* Los programas analizadores sintácticos de XML, que se construyen en una com pu ta do ra dada, no re quie ren del men cio na do des crip tor del tipo de do cumento (DTD) para poder separar un documento en sus componentes.

* Nopermiteninguna des via ción delasintaxis es tán dar. Esto quie re de cirque todos los documentos codificados en XML pueden ser editados, guardados y enviados sin importar el programa analizador de XML del receptor.

* Los do cumentosXML pue den proveer hojasdeestilo que le per mi ten a los navegadores (Netscape, Explorer, etcétera) convertir los documentos para presentarlos en HTML en la pantalla.

* Losanalizadores XMLpuedenanalizarinclusodocumentosHTML bien formados. Esto permite que la transición de documentos ya existentes en HTMLen una em pre sa que esté evolu cio nan do haciaXMLpueda ha cersede manera gradual y programada.

Como ya se ha establecido, XML es un metalenguaje que permite establecer un conjun todereglasparade finirunasin taxises pe cífica,lacualseráaplicada solamente enunescenario preestablecido.Losdocumen tosquepuedenespecificarseentonces bajo XML pue den ser de muy diversas na tu ra lezas, por ejem plo las pro pias del mundo delas finanzas, al es pe ci ficarda tos de ac cio nes, co tiza cio nes, tipos de cam biode divisas, etcétera;olos da tos de tipobibliográfi cocon teni dosenun documen to dado; o bienda tos de com pues tos quí mi cos den trode una fór mula; o pla nos y da tos sobrelaconstruccióndeunavión,etcétera.

Sehan presentadounase riedepostuladosacercadelas principales diferenciasentre el es tán darHTML y el XML. Tal vezla me jor ma ne ra de com pren der estoplenamente 
sea con unpequeñoejemploqueloilus tre. Tomemosunmíni mo trozodelguión dra mático DonJuan Tenorio, tal como apa re ce el tex to im pre soen unlibro (sin len guaje de marcadoelectrónico):

ACTO I

Escena 1. Don Juan, con antifaz, sentado a una mesa escribiendo; Ciutti y Buttarelli a un lado es pe ran do. Alle van tar se el te lón se ven pa sar por la puer ta del fondo máscaras, estudiantesypuebloconhachones, música, etcétera.

Don Juan: ¡Cuán gritan esos malditos!

¡Pero mal rayo me parta

Si en concluyendolacarta

No pa gan caro sus gritos!

Buttarelli: A Ciutti

Buen carnaval

Ciutti: $\quad$ A Buttarelli

Buen agosto

Para rellenarlaarquilla.

Paraleerein terpre tareste tex to deZorrilla, unaperso na no re quiere marca do adicio nal. Es ca paz de re co no cer el sig ni fi ca do de cada una de las partes que lo for man basándose en suexperienciay el simple “marcado”tipográfico.

La versiónHTMLde estetex to severíaasí:

$<\mathrm{H} 1>\mathrm{ACTO} \mid</ \mathrm{H} 1>$

$<P><\mid>$ Escena 1. Don Juan, con antifaz, sen ta do a una mesa es cribien do; Ciuttiy

Buttarelli a un lado es pe ran do. Alle van tar se el te lón se ven pa sarpor la puerta

delfon do más caras, es tu dian tes y pueblo con hacho nes, música, etcéte

ra. $<||>\langle P\rangle$

$<\mathrm{P}><\mathrm{B}>$ Don Juan: $</ \mathrm{B}>$ ¡C Cuán gritan esos malditos!

¡Pero mal rayo me parta

Si en concluyendolacarta

No pa gan caro sus gri tos! $\langle/ \mathrm{P}\rangle$

$\langle\mathrm{P}\rangle\langle\mathrm{B}>\mathrm{Buttarelli}:<\mid \mathrm{B}\rangle \quad\langle|>\mathrm{A}$ Ciutti $<||>$

Buen carnaval $\langle\mid \mathrm{P}\rangle$

$\langle\mathrm{P}\rangle\langle\mathrm{B}\rangle$ Ciutti: $\langle\mathrm{B}\rangle \quad\langle|>\mathrm{A}$ Buttarelli $<||>$

Buen agosto

Para rellenarlaarquilla. $<|\mathrm{P}\rangle$

LanomenclaturademarcadoHTMLeslasiguiente:

$<>$ encierra el principio de una etiqueta de marcado.

$</>$ encierra el fin de una etiqueta de marcado. Siempre van por pares: $<>$ comienza marcado; $</>$ termina marcado para esa etiqueta. 
H significa "Header" o encabezado. Puede haber más de uno y por tanto se numeran: $\mathrm{H} 1$ es el "Header 1". $<\mathrm{H} 1>$ marca de comienzo del encabezado 1. $</$ H1 $>$ marca del fin del encabezado 1 .

$\mathrm{P} \quad$ significa "Paragraph" o párrafo. $<\mathrm{P}>\mathrm{y}</ \mathrm{P}>$ indican el principio y el fin de un párrafo.

I significa "Itálicas". "B" significa "Bold", negritas. Puestos en pares para indicar dónde comienza y termina esa característica del texto, con ayuda de la diagonal " $/ "$.

La ver siónXMLdeeste mis motex topodríaser:

$<$ ACT $><$ TITLE $>$ ACTO $\mid</ T$ TLE $>$

$<$ SCENE $>$ TITLE>Escena 1. Don Juan, con antifaz, sen ta do a una mesa es cribiendo; Ciutti y But ta rellia un lado es pe ran do. Alle van tar se el telón se ven pa sar por la puerta del fondo máscaras, estudiantes y pueblo con hachones, música, etcé tera. $\langle T$ TITLE $>$ $<$ SPEECH> <SPEAKER> Don Juan:</SPEAKER> $<$ LINE>

¡Cuán gritanesosmalditos!

¡Pero mal rayo me parta

Si en concluyendo la carta

$</ L I N E>$

No pagan caro sus gritos!

$</$ SPEECH $>$

$<$ SPEECH $>$

$<$ SPEAKER>Buttarelli:</SPEAKER>

$<$ LISTENER>A Ciutti</LISTENER>

$<$ LINE $>$ Buen car naval </LINE $>$

$</ S P E E C H>$

$<$ SPEECH>

$<$ SPEAKER>Ciutti:</SPEAKER>

$<$ LISTENER>A Buttarelli</LISTENER>

$<$ LINE>Buenagos to

$</$ SPEECH $>$

para rellenarlaarquilla. </LINE>

Lasterminaciones delosmarcadores $</$ SCENE $>\mathrm{y}</$ ACT $>$ estaránhastadonde seencuentreelfinaldelaes cenay elfinaldelacto, portantonoseescribenaquí.

Losmarcadores $<>y</>$ (abriryce rraretique ta) se ma nejanigualque enHTML y porpares.

Si se ob servan es tos ejem plos marca dos se pue de no tar que en el primer caso, con HTML, los marcadosnosindican en cabezados, párra fos, itálicasynegritas; es decir, indicaciones paraeldes pliegue del tex to, peronada sobrelas par tes oel con tex to 
quevan compo nien dola obra. En el se gun do ejem plo, el deXML, pue deobservarse que se ha marca do, ade más del en ca be za do o título, dón de em pieza el acto I; dón de empiezala es cena; dón de em piezay ter mina eltítulo; dón de comienzay terminacada diálogo(speech); quién es el ac tor del diá lo go y quién es el que es cu cha, y cuál es la lí nea del diálogo que le pertenece a cada quien. De esta forma, el documento ha quedado marcadoconuna se rie mu cho más comple tade elemen tos, la cual es sus ceptible de ser analizadanosóloporlossereshumanos sinotambién porunacomputadora.

Supongamos que queremos que la computadora reproduzca por medio de un progra masin te tiza dor de vozlos diálogos a tra vés delas bo ci nas de la mis ma; porsupues to con vo ces apro pia das para cada perso naje. Con elprimermarca do, elHTML, se ría muy di fícil lo grar lo; con el se gun do, la má qui na pue de, al ir "le yen do" el tex to, identificar fácilmente quién es el actor que habla $<$ SPEAKER $>$, y utilizar siempre la misma voz para ese personaje, y reproducir sólo el texto que se encuentra en $<$ LINE $>$, ignorando para el parlamento las indicaciones de escena, título, nombre delactor,quiénessuinterlocutor,etcétera.

Apropó sito hemosdeno minadolas etiquetaseninglés: title, act, speech, liste ner, line, etcétera. Podríamoshaberlasdenominadotítulo, acto, diálogo, es cu cha,línea, etcétera. Sería válido y funcionaría en nuestro ámbito. Pero ¿Qué pasaría si ese diálogo quie re ser pues to en la In ternetpara su uso universal? ¿Cómo sa bránlas má qui nas de otras par tes del mun do que < diálogo $>$ es eso, un diálo go? Sibien no hay un es tán dar es tablecidoen cómodebella marse a una etique ta, es muy probable que en poco tiempo, los dramaturgos que quieran poner sus textos en esta forma se inclinen por un nom bre uni ver sal para la mar ca, y lo más pro ba ble es que sea un nom bre en in glés y que, por convención, todo el mundo acepte en poco tiempo que " $<$ speech $>$ ", por ejemplo,essiempreundiálogo enuna obradeteatro.

Otro ejem plo de aplica ción de este marca do es que una com pu ta do ra, bajo pe di do, pue de ha cer fá cil men te una lis ta de los per so na jes de la obra, $<$ SPEAKER $>$ y ponerla en su di rec to rio de pa la bras para re cu pe ra ción, conobje to de que unusua rio en la red bus que y en cuen tre a tal o cual per so na je que apa re ce en una obra. Hacer esto conelprimermarcadoseríasumamentedifícil.

Cabehacernotaraquíqueenambosejemplos no he mos utilizado to doslos elementos posibles del marcado de cada metalenguaje.Ni con mucho hemos agotado todas lasetiquetas utilizables. Porsupues to, en XMLpue den agre garsemarcas para tipogra fía, pero nosotros sólo hemos seleccionado unas cuantas, simplificando el ejemplo, parailus trarelconcepto. Noes el propó sitodeestedocumentopresentartodosloselemen tos de marcado de cada for ma to, por que esto se ría in men so, pero síexis ten tex tos quehacen esto.Laideaes resal tarlascaracterísticas des critasalolargodeestetexto.

\section{Resumiendo,podríamosafirmarqueXMLes:}

Simple - La especificación completa mide menos de 30 cuartillas. XML ha sido diseñado para facilitar aún más la escritura de programas con respecto a HTML o SGML. 
Extensible - Cada quien puede inventar sus propias etiquetas para marcar cualquier tipo de documento, y ser éstas compartidas. De hecho, XML es un metalenguaje que le permite al usuario definir su propio lenguaje de marcado.

Un estándar abierto - XML es SGML. Ello significa que no es necesario saber programar; existen muchas herramientas eficientes que permiten ya sea crearlo, manejarlo o implantarlo en una computadora y distribuirlo.

Eficiente - XML tiene entes interconstruidos para reutilizar fragmentos de documentos, así, estos sólo tienen que ser transmitidos una vez.

Basado en la experiencia - XML ha sido diseñado por personas que tienen amplia experiencia en los lenguajes de marcado y han capitalizado las enseñanzas que el uso de ellos les ha suministrado a lo largo de los años.

Consensuado - El diseño de XML incluye los puntos de vista de los organismos coordinadores de HTML y SGML, así como los de personas que han desarrollado importantes aplicaciones con estos estándares.

Libre - Nadie tiene la propiedad o patente de XML, ni podrá tenerla, ya que tanto SGML como XML han sido definidos como estándares internacionales. Por lo mismo su uso o desarrollo no implica el pago de ninguna regalía.

Internacional - XML tiene interconstruido un soporte para textos en prácticamente todos los alfabetos del mundo, incluyendo técnicas para consignar el lenguaje y/o código del alfabeto utilizado.

Listo para ser usado - Los "browsers" o navegadores del web, en sus últimas versiones, son capaces de leer especificaciones XML. Los hipervínculos, textos y multimedios pueden ser vistos tal como se hace ahora con un documento HTML.

Manejable - XML incluye métodos para declarar y reforzar las estructuras documentales usadas actualmente, como las de bases de datos.

Validable - XML tiene técnicas que permiten la validación de los documentos involucrados, así que uno puede estar seguro de que los documentos registrados con él son creados correctamente.

\section{ANÁLISIS DE LA PROBLEMÁTICA}

Parapo derpro ce deraun análi sis crítico dela si tuación es ne ce sa rioprimeroacotarelentornoenelcualdeseamosrealizarlo.

Sindudaunadelascaracterísticas delestablecimientodecoleccionesybibliotecas digita les es queés tas no se limitan sólo a ma te ria les de re fe ren cia, sino, cada vez más, a textos completos. Las bibliotecas digitales tratan ahora de establecer los mejores mecanismos para almacenar estos textos completos electrónicos de tal forma que sean totalmente recuperables y explotables por las comunidades académicas, más alláde ha ceruna sim plebúsque da de pa la bras en el tex to yopera do res booleanos. Ha quedado demostrado que XML tiene un gran número de ventajas nativas para este 
pro pó sito, y que por ello mis mo será ellen guaje de marca do más utiliza do en esta déca da has ta que sea sus ti tui do poralgo mejor. No obs tan te, su mis ma universalidady exten sibilidad,ylasmayo res ventajas deXML, in troducenuna se riedeproblemasen elentornoenqueéstepuedeserutilizado.

En efec to, de be mos em pe zara aco tar elproble ma en nues tro me dio; como se ha mencionado XML pue de seruti li za do para des cribir toda cla se de do cu men tos: des delos rollos del MarMuer to, pa san do porunex pedien temédico o un libro, has talas especificaciones de un avión supersónico. Puede serusa do en tonces enlos sec tores educativo, delaconstrucción, médico,gubernamental,etcétera.Cadasectorpuedey debería empezar a sentar las ba ses para de fi nirsus do cu men tos más re le van tes. De hecho, puede existir más de una de fi nición para do cu men tos de un mis mo sec toro tipo, lo que ha ría muy di fícil su ma nejo por parte de usua rios dis tin tos a aque llos para loscualessehayadiseñadolainformación.

Poreste mo tivo enla ac tua lidad se es tán de finien do des crip to res de tipo de documento (DTDs) por grupos sectoriales con intereses afines, de forma que están surgien doestán daresavalados pororganis mos quega ran tizanquecualquierusua rioque losadop te como suyos, traba je conlas mis mas etiquetas eidén ticasnor ma tividades, como se hace con el actual HTML. Como ejemplos de esto tenemos CML, Chemical MarkupLanguagepara el sec tor químico,MathML, MathematicalMarkupLanguagepara definirdatosmatemáticos, SMIL,SynchronizedMultimediaIntegrationLanguage, para definirpresentaciones en re cursos multimedios, etcétera. A estostiposde va riantes de documen tos selesconocecomo clasesyesuntérminomuyimportante.

De he cho para un mis mo tipo de do cu men to pueden exis tirdiversos en fo ques y necesidades. Tomemos el caso del documento "libro". La definición de este documento obedecerá a los intereses particulares de determinado sector. Por ejemplo, para al guien que co mer cia li za li bros en la Web, su de fi ni ción de do cu men to sóloincluiría elementosmínimosdeidentifica ción: au tor, título, año, editorial, ISBN; pero también datos tales como precio, descuento, peso, costo de envío, disponibilidad y/o tiem po de en trega, con di cio nes de pago, et céte ra. Poco quevercon el ambien te de una biblio te ca, en don de fal tan mu chos otros da tos con fi nes de re feren cia de los documentos, mientras que algunos otros de nuestro ejemplo salen sobrando. Si el documento por de finirse es un tex to com ple to, deben agregarsemu cho más da tos. Porellolasdefinicioneshechasparaunsectorseríaninútilesparaotro.

En elmedio aca démico,y en particulareldelas bibliote cas, es ne ce sarioen tonces comenzaraestablecerlas definicionestipo paracadaclasededocumento, conobjeto de satisfacer cabalmente las necesidades de consulta por parte de sus comunidades de usuarios. Cabe recordar que existen además distintos tipos de bibliotecas, y por ende, deberá haber ciertos "matices" en estas definiciones. Estos estándares deberían, porsupues to, es taracordes con nues tra realidad mexica nay con lascaracte rís ticasdenuestraproduccióneditorial,entornohistóricoycultural,idioma,etcétera.

Debemosreflexionarentoncessique remossentarnosaesperarqueestas de finiciones sean he chas porotros en tornos ex tranjeros, o porsec to res aje nos ala aca de miaya 
labiblioteca, oporpersonal no pro fesional en el regis tro do cumental; o sies el medio bibliotecario académico el que debe comenzar a sen tarlas ba ses para es ta ble cerestán da res en es tas de fini ciones, como ya lo han co men za doa ha cer otros sec to res en otras par tes del mun do.El sectorbibliotecarioes elquedebe ríahacerlas es pe ci ficacio nes delos do cu men tos para elme dioacadémico, y se ría ideal que fue ra el sectorbibliotecariomexicanoelquelohicieraparaelmedioacadémicomexicano.

Hemosvis toya eldes pertary elaugequees tán to mandolas bibliotecas digitalesen todo el orbe, y el que co mien zan a te neren nues tro país. Em pe za mos a ver ya al gu nas colecciones de documentos digitales. Sin em bargo, cabe ob servarque casi to das estascolecciones están presentadas enforma to HTML y que casi ninguna de estas colec cionesde biblio te cas digitales tieneyasusde finicionesXML, aun que hay que destacar que algunas ya lo están considerando y preparándose para ello. Se venden o dis tribuyen ya en el me dio al gu nos progra mas o aplica cio nes que se anun cian como "generadores de bibliotecas digitales", tanto de origen nacional como extranjero. Empiezanasurgiryalasprimerasbibliotecas digitalesconespecificacionesXML. La pregunta crucial es ¿quién crea esas definiciones para los tipos de documentos que manejan esas biblio te cas? Unexa men más de talla do nosen seña ráqueporlogene ral no han sido he chas porper so nal pro fe sio nal en re gis tro do cu men tal, y porlo tan to cuandomuchorepresen tan elpun to devis tadeun sólopro fe sionalosector.Las "etiquetas" de marca do mu chas ve ces son sólo réplicas de las es ta ble ci das porMARC o AACR2, quesonbuenasparamaterialdereferen ciaperonopara tex tos completos.

Caberesal taraquíque es tas de ficien ciasno seha cen no tardeun modo peyorativo alguno. Las personas u organizaciones que están detrás de ello han hecho su mejor esfuerzo con los elementos disponibles hasta este momento y sin ningún estándar prestablecidoyporterritorios noexplorados. Simplementede seamos re saltarelhecho y el ries go que se co rre de crearuna Ba bel al re de dor del tema, has ta que los me jo res están da res de sa rrolla dos sevayan fil tran do en elmedioyla situa ción se estabilice. Pero ello puede tomar mucho tiempo y costar caro, además de consumir recursos que en nues tro país no so bran y de ben seruti li za dos ra cio nal men te para el me jor de sarrollo de nuestrasbibliotecas y colec ciones digitalesy, porende, delas co munidadesacadémicasalasqueéstassirven.

Esaltamentenecesario,entonces, comenzaraes tablecerlosestándaresmínimosque es tas de finiciones deben contenerparacada tipodedocumen to orien ta do ha ciaelsector académicoengeneralybibliote carioen particular, con sideran donosólolas ca racterísticas de referencia sino los tex tos completos,yenfocándoloa nuestromediomexica no, a nuestrasbibliotecasyanuestroentornohis tórico, social, económico, etcétera.E s tas definicionesdebenserefectuadasporpersonalexperto,profesionalymul tidisciplina rioen ta reas de re gis tro, re cu pera ción y dis tri bu ción do cu men tal, con el fin de que sean avaladasporlas bibliotecas digitalesque seestán creandoyadop ta das porellas como suyas. La idea escrearun ambiente deho mogeneiza cióny calidad en los regis trosylas colecciones queseformen. 
Nosetra tadeponerle unaca misade fuerzaalases pecificaciones delos documentos, sino de crear una definición de calidad sobresaliente como base que pueda ser aceptada con con fianza porlas biblio te cas digitales de nues trome dio, para que a partirdeellacadabibliotecapuedaseguirconstruyendosuspropiasespecificaciones.

¿Quéesloquehabríaquedefinirentonces?

\section{ALCANCES DEL ESTUDIO}

Típicamenteundocumento XML tiene dos partes: una de finición dellengua je que se usa, y el contenido del documento en sí mismo. La definición puede ser escrita usan do una DTD (de fi nición o des crip ción del tipo de do cu men to), que es una sin taxis para des cribiren for ma deHTML. Esta DTD pue de es tarin mersa en el pro pio documento o ser externa a él. Al ente descrito se le llama objeto. Esta técnica ha sido usa dahas taestemomen to congranin ten sidadyéxito, dadalarecien teliberación del estándar XML. Deini cio, se an toja ría que el pro yec to de be ría ten dera de sa rro llarlas especificacionesdetalladasmencionadas anteriormenteenformade DTDs que puedenserdistribuidasparalograrelpropósitoestablecido.

Sinembargoenlosúl timosmeses sehavenidode sarrollandouna formamáseficazpara de finirdocumen tos bajoeles tán darXML, conocida comoes que mas de tipo de elemento osimplemen teesquemas, que de finenlas caracterís ticas delasclases deobjetos. Es tos esquemas pueden de finirse comounDTD que per mi teademás supropia am plia ción median teun me talenguajepro pio crea do al efec to, que de fine a suvezalDTD. Di cho de otra forma, el esquema representa el metadato para un documento XML asociado, y describe quépue dey quéno pue de serin cluido enun do cumen toXML [Campbell, 2003]. Con esta técnica pueden desarrollarse vocabularios especiales para definir y documentar clases de objetos. Esta técnica promete mayores y mejores alcances para definir documentos y se perfilacomomásinteresantecomoproyectodeinves tigacióndefrontera. Elpresente trabajopreten de ex plicarloque es unes que maXML y de des cu brir las ven tajas más allá de un DTD. [Young, 2002]

Va mosa tra tardeirilus trando esto, para empezar ¿cómo se ve un es que ma de tipo deelemento? aparentementenodi fieremu chode undescrip tor DTD. Lo me jor será ilustrarlo con un pequeño ejemplo. Todas las declaraciones de un esquema están contenidasenun elementodeesquema comoen el siguien te ejemplo:

\section{ESQUEMA DE TIPO DE ELEMENTO}

$<$ ?XML ver sion='1.0' ?>

$<\mathrm{s}$ :schema id='EjemploEsquema'>

$<!-$ el esquema va aquí. $\rightarrow>$

$</$ s:schema $>$ 


\section{Declaración del Tipo de Elemento}

El nú cleo de un es que ma de dato XML es la declaración de Tipo de Elemento, la cualde fine una clase deunobjeto. El atributo id tienela doblecaracterís ticadeiden tiff carlade finiciónydenombraruna claseespecífica.Losdescripto res básicosdenomencla tu raya es tán de finidosen XMLy es tán en in glés. A esto se le de no mina el do mi nio namespaces en XMLy esuna ta bla ya de fini da, sibien en cons tan te ac tualiza ción. No deben confundirse los descriptores básicos de nomenclatura (string, integer, maxxocur, etcétera) yaes tablecidos, conlos nombresposibles delasetique tas.Ésaes precisamentelaven taja de XMLso breHTML oMARC:los des crip to resno es tán es tableci dos yuno pue de bau tizarlos comounoconside repertinente.Sibien podría mos bau tizaratodos ycada unodelos elementos enlos descrip to res encastellano: au tor, título, tema, etcétera, en una sana de fen sa denues tra len gua, ello no será con ve nien te en la práctica, porque esta de fi ni ción que da ría sólo para uso lo cal en los países de ha bla his pa na, y como lo que se desea es que es tas de finiciones sean deíndoleinterna cional, talvezlo mejor sea ha cerloenin glés. No obs tan telo an te rior, es po sible es table cer "alias" delos nombres, yes tos alias pue den ser las no men cla tu ras en cas tella no, con locual pue den utilizarseambas no men cla tu ras agregan do unas cuan tas de finiciones más y cuidan do que exis tan simul táneamen te.Enlos ejemplos sub secuen tesutilizarédes cripto resenespañolpararesaltarlafacilidadconqueunopuededenominarlos.

Para en ten dercómo se cons truyeun es que ma debe mos en ten der pri me ro cómo se establecen las definiciones de los elementos. Hagamos unos ejercicios sencillos previos de cómo sede fine en XML. Utilice mos para el ejemplola de finición de un elemento "autor" dentrodeundocumento"libro":

<ElementType id="autor"/>

Dentrodel tipodelelemento, po demosincluirun subelementodescripción (description), para que las perso nas pue dan des ci frarcuáles el con tenido de ese ele men to. Dehechopodemosagre garmúltiples subelemen tosaunelementoconobje todedejarloperfectamentedefinido.[Thompson,1997]

$<$ ElementType id="autor"/> <description>La personaoinsti tuciónqueescribióla obra.</description> $<$ /ElementType $>$

\section{Propiedades y Contenidos}

DentrodelElementTypepodemoscrearsubelementosquede finanlascaracterísticasdeloahícontenido.

$<$ ElementType id="autor">

<string/>

$</$ ElementType>

$<$ ElementType id="libro">

$<$ ElementType="\#autor" occurs="ONEORMORE">

$</$ ElementType> 
Este ejem plode fine dos elemen tos, "au tor" "y "libro". In dicaque un ele men to "libro" pue de teneruno ovarios elemen tos "au tor". Elelemento au tor pue de contener una ca de na (string) de ca racte res. Den tro de un tipo de ele men to pue den es tar con tenidos varios subelementos (element, group, any, empty, string, etcétera). Éstos indican cuáles pue den serin clui dos en ese tipo de ele men to ein clu so su se cuen cia. Los tipos de elementos tienen también un atributo ocurre (occurs), el cual puede tener cuatro valores:requerido "required”, opcio nal "optional”, cero o más "zeroormore”, y uno o más "oneormore".

Siguiendoestasreglas, podemoscontinuarconstruyendoestadescripción: <ElementType="\#titulo" occurs="OPTIONAL">>

Podemos agregar también unos su belementos en "grupo"; diga mos "prólogo"e "introducción", los cuales pueden venir en el documento libro. Cabe hacer aquí la aclaración de que estamos definiendo un documento para ser ingresado en texto completo,ynosóloloselementosdesufichacatalográfica.

Un re gis tro más ela bo ra do (sin pre ten der serex haus tivo) y sólo a ma ne ra deilustración, podríaversedeesta forma:

$<$ ElementTypeid="libro">

$<$ ElementType="\#autor" occurs="OPTIONAL"/>

$<$ ElementType="\#titulo" occurs="ONEORMORE"/>

$<$ ElementType="\#fecha-de-copyright" occurs="OPTIONAL"/>

<groupocccurs="OPTIONAL">

<elementType="\#prefacio"/>

$<$ elementType="\#introduccion"/>

$</$ group $>$

$<$ ElementType="\#ISBN" occurs="OPTIONAL"/>

$<$ ElementType="\#texto-del-libro" occurs="ONEORMORE"/>

"ElementType="\#para-edades" occurs="OPTIONAL"/>

$</$ ElementType>

Undocumentomarcadoacordeconestaestructurapodríaverseasí:

$<$ libro>

<autor> Miguel de Cervantes Saavedra </autor>

$<$ titulo> El IngeniosoHidalgo Don Quijote de la Mancha

$</$ titulo $>$

$<$ prefacio>Aquíiríaun prólogoescrito......</prefacio>

<introduccion> Aquí iría una introducción.... </introduccion>

$<$ isbn $>123-456-789-0<$ <isbn>

$<$ texto-del-libro> En un lugar de la Man cha, de cuyo nom bre no qui sie ra

acordarme.... (sigue todo el texto del libro)

$<$ /texto-del-libro>

$<$ para-edades> todo público</para-edades>

$</$ libro $>$ 
Uno delos as pectos de mayorim portan ciaen la de finición del es quema, con sis te en que, como ya habíamos menciona do, pue den es tablecerse "alias" delos descriptores. Un "alias" consiste, como su nombre lo indica, en un segundo elemento de identificación de un nombre de elemento. Su importancia radica en que estos alias pueden ser usados para tener una equivalencia en castellano de cualquier nombre definido previa o posteriormente. El descriptor se llama elementTypeEquivalent.

<elementTypeEquivalent id="libro" type="\#book"/>

$<$ elementTypeEquivalent id="autor" type="\#author"/>

<elementTypeEquivalent id="titulo"type="\#title"/>

$<$ elementTypeEquivalent id="personaje"="\#personaje"/>

...etcétera.

De esta for ma, den tro de la de fi ni ción del do cu men to, "li bro" y "book" se vuelven si nó ni mos y pue den serusa dosin dis tintamen te den tro dela de fi ni ción. Así, conestetipode elementos, podemosmantenerlainterna cionalidad dela de finiciónyseguirmanejandolostérminosennuestralengua.

Aho rabien, este fue sólo un pe que ño ejem plo rela cio na do conlos atribu tos delas primerasversiones de XMLpara fa cili tar su com pren sión, pero su uso pue de irse expandiendo y sofisticando hasta dimensiones inimaginadas. Hemos definido un elemen to "au tor" forma do de una sola ca de na de ca rac te res, pero ello no tie nepor qué limitarse a eso. Po de mos de finir au torcomo unase rie de ele men tos, digamos, nombre, apelli dos, título o cargo, fe chas dena ci mien to y muer te, seu dó nimos, etcéte ra. Suena más parecido a lo que es ta mos acos tum bra dos a ver en las re glas an gloamericanas de catalogación AACR2. Igualmente podemos subdividir título y subtítulo, y piedeimprentaenciudad,editorialyaño,etcétera.

Perola de fini ción no seque da en los ele men tos de la ficha ca ta lográ fica. Como se havis to po demos des cribirel tex to comple to de unlibro. Po demos subdividirlo en capítulos, secciones, apartados, etcétera.Podemosseñalarnotasapiedepágina, citas bibliográficas, palabras a ser referidas en un glosario, direcciones de otras páginas web, etcétera. Podemos marcar personajes, escenas, lugares, diálogos expresadoso pensamientos del personaje, etcé te ra, como ya lo he mos mos tra do en otros aparta dosprevios.

Podemos también, agregando algunas técnicas llamadas API (Application ProgrammingInterface), marcardi fe ren tes valo res para al gúnele men to; es decir, pode mos predetermi narsieseele men to es una ca dena de ca rac te res, un nú me ro en te ro o fraccionario, ein clu so con tarcon rangos po sibles; una fe cha en al gún forma to dado (ISO proporcionavarioses tán dares), unbooleano, unelemen to que debe exis tir deacuerdoconunatablaocatálogopreestablecidos, etcétera. 


\section{CONSTRUYENDO UN PRIMER ESQUEMA}

Una vez que hemos hecho una pequeña presentación de un esquema podemos pa sara cons truir un es que ma ve rí di co tal como pue de sercons trui do en la vida real. Para poder iniciar esta construcción partiré de un ejemplo real de un descriptor de tipodedocumen to (DTD) para un libroy de ahícon tinua récon la cons truc ción deun es que ma.Los nombres delos atributos delos tags oetique tas es tán to ma dos delXML namespaces para fines deuso ya normalizado, deacuerdoconlo es peci fica do porXML. Parainiciarel ejemploutilizaréla obradelTeno rioya presen ta da conanterio ridadsegún el or den de Van der Vlist [Van der Vlist, 2001]; de acuer do con un DTD ya es tablecidopodríaverseasí:

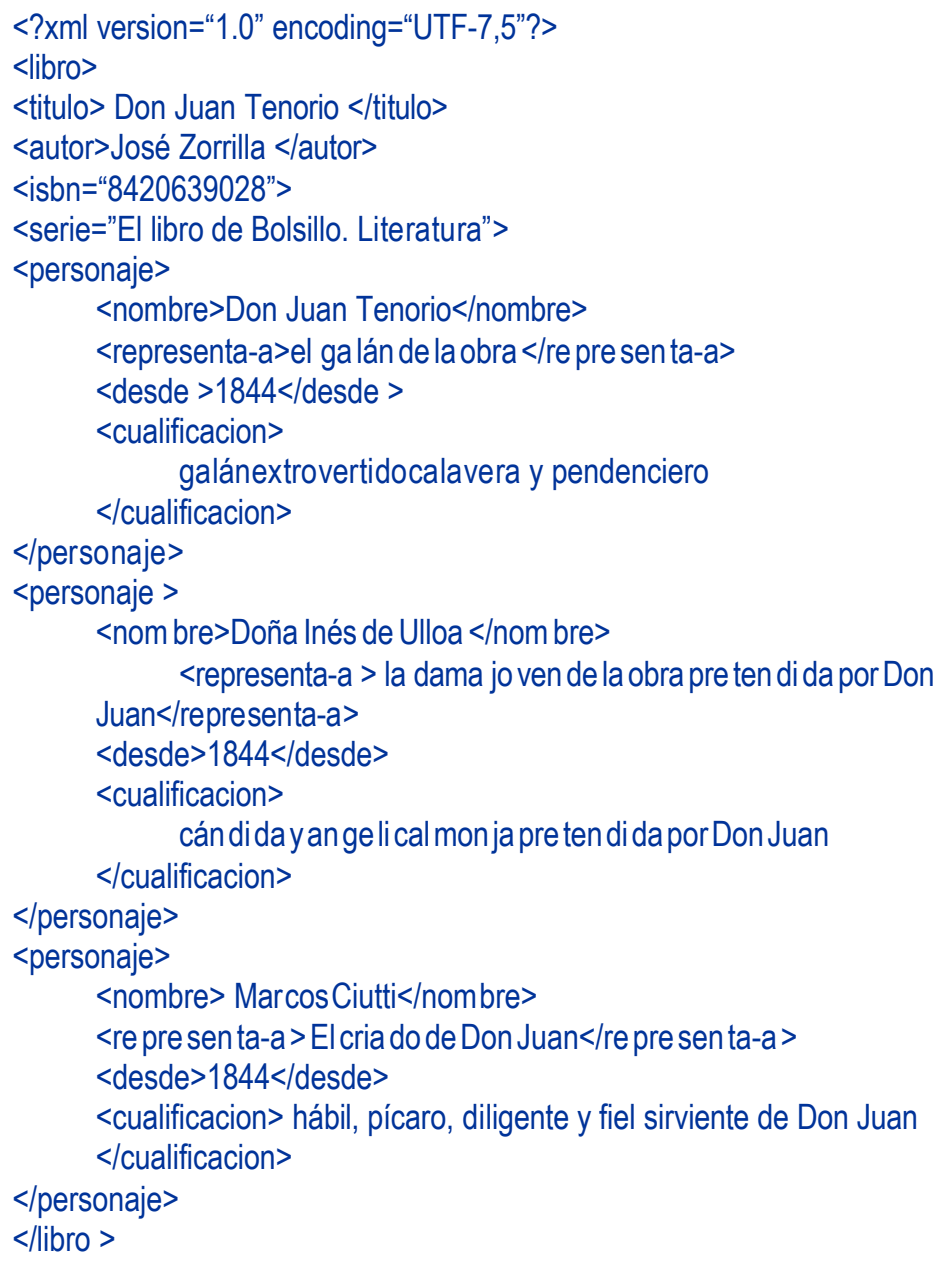


Para es cribirun es que ma que des criba los do cu men tos marca dos en esta forma, simplementeseguiremossuestructuray ele men tos con formelos vamosencontrando;para empezar,abrimoselelementoxs:schema:

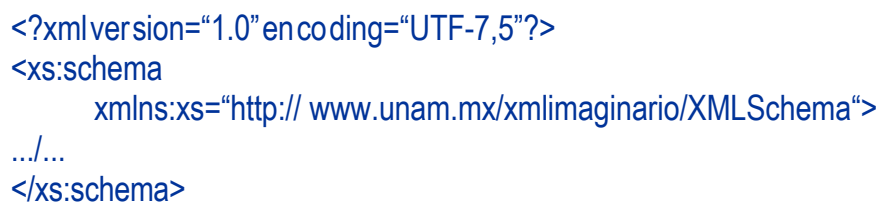

La di rec ción http in di ca da es don de debe re si dir el es que ma; en este caso es fic ticia. Sirve para abrirnues tro es que ma y tam biénguar da la de fi ni ción delnamespace desti no y va rias opcio nes que se asig nan poromi sión, al gu nas de las cuales se ránex plica dasmásadelante.

Para cuadrar la etiqueta inicial para el elemento "libro", definimos un elemento con ese nom bre. Este ele men to tie ne atribu tos e "hijos" no- tex to, porlo queloconsideramos como de tipo complejo complexType, dado que los otros dataTypes como simpleType es tán re serva dos para ti pos de da tos que con tienen sólo valo res pre de ter minadosynoelementososub-nodos de atribu to.Lalis tade"hijos"delelemento "libro" esdescritaporunelementodesecuenciasequence:

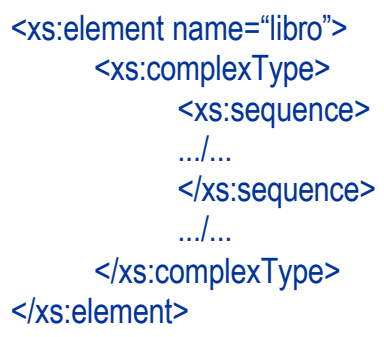

La se cuencia es una "guía" que de fine una se cuencia or de na da de su be le men tos. Veremosotrasguías, choice and allenlas siguientessecciones.

Podemos de finiraho ralos ele men tos de au tory título comotipos sim ples, notienenatribu tos ohijos no- tex toy pue den serdes critos directamen te como elementos. El tipoxs:string (cadenadecaracteres)losde finedeesa forma:

$<x s: e l e m e n t ~ n a m e=" t i t u l o "$ type="xs:string"/>
$<x s:$ :element name="autor" type="xs:string"/>

Ahoraes tablecemos el elemento "personaje", elcuales de tipo complejoydes cribe a los diferentes personajes de la obra. Nótese cómo se define su cardinalidad, es decir,elnúmerodevecesquepuedeocurrir: 


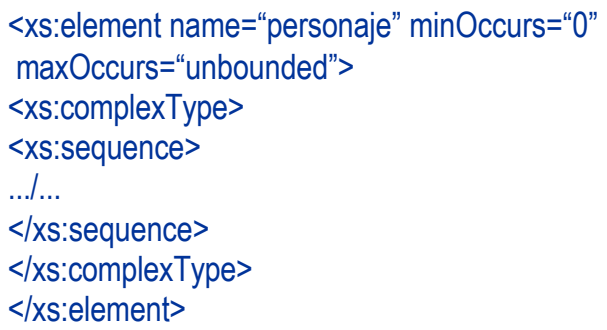

Especificamoslalistadetodossus“ hijos”delamisma forma:

$$
\begin{aligned}
& <x s: e l e m e n t \text { name="nombre" type="xs:string"|> } \\
& <x \text { :element name="representa-a" type="xs:string" } \\
& \text { minOccurs="0" maxOccurs="unbounded"/> } \\
& <x \text { s:element name="desde" type="xs:date"/> } \\
& \text { <xs:element name="cualificacion" type="xs:string"/> }
\end{aligned}
$$

Terminamos su descripción cerrando el complexType, y los elementos element y sequence.Podemosahoradecla rarotrosatributos deloselementosdeldocumento:

$<x$ :attribute name="isbn" type ="xs:string"/>

$<x$ s:attribute name="serie" type ="xs:string"|>

Ysecierranloselementosrestantes.

Este dise ño, co no cido en el me dio como de tipo matriushkea por las mu ñe cas ru sas de ma de ra que se ani dan una den tro de la otra, si gue de cer ca la es truc tu ra del do cumen to mues tra. Una delas ca rac te rís ti cas cla ve de tal diseño es la de de fi nircada elemento y atributo dentro de su contexto permitiendomúltiples ocurrencias del mismoelementoparaobtenerdiferentesdefiniciones.

Listacompletadelprimerejemplodelesquema:

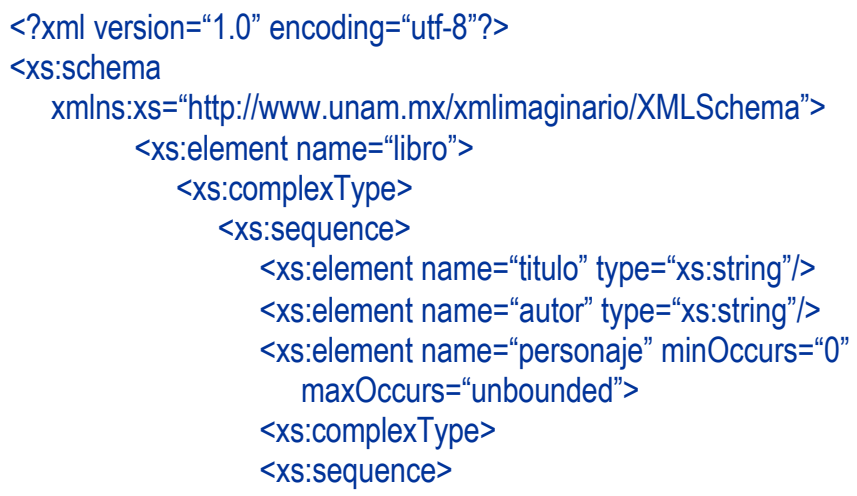




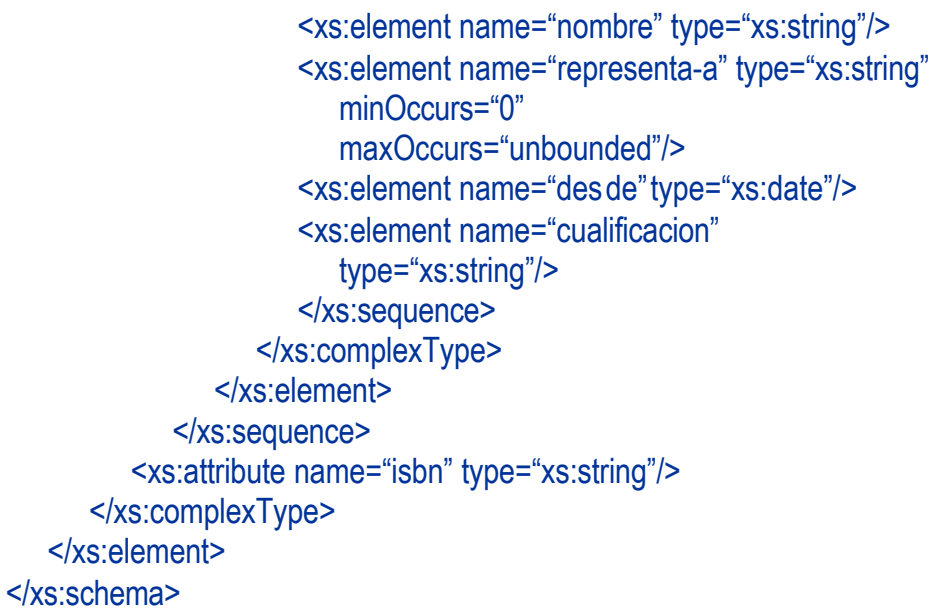

Nóte secómoelprimerdocumentodes critocon marcadoresXMLcorrespondea un do cu men to, la obra del Te no rio de Zo rrilla en par ti cu lar. Esteúl ti mo lis ta do co rresponde al es que ma de ese do cu men to; es de cir, con este es que ma po de mos descri bir to dos los do cu men tos u obras que ha yan sido marca das con el es tilo del do cumento presentado. Podemos entonces procesar los documentos descritos en esa forma, pero si anexamos el esquema a los documentos los sistemas pueden saber cómofueronconstruidos esosdocumentos. [Morrison.2002]

\section{DIVIDIENDO EL ESQUEMA}

Si bien la estructurapresentada anteriormente es muy sim ple, pue delle vara muchos anida mien tos delas de fi ni cionesinmersas, ha cién do la muy difícildeleery difícil de mantener cuan do los do cu men tos son muy com ple jos. Tie ne tam bién la desven taja desermuy diferentea una es truc tu ra de unDTD, lo cuales un obs tá culo tan to paralas personas como paralos programas quedesean trans formarun DTD en un esquemaXML.

Un segundo diseño que presento está basado en una "tabla plana" de todos los elementos disponibles en un documento, y para cada uno de ellos, listas de los elemen tos "hijos" y sus atri bu tos. Este efec to se lo gra a tra vés deluso de re fe ren cias hacia los elementos y definiciones de atributos que requieren estar dentro del ámbito delreferencista, yseven enforma deunatablaplanacomoyamencionamos.

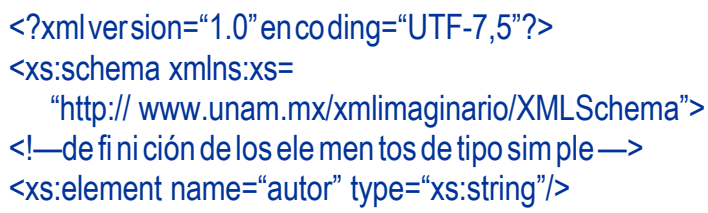




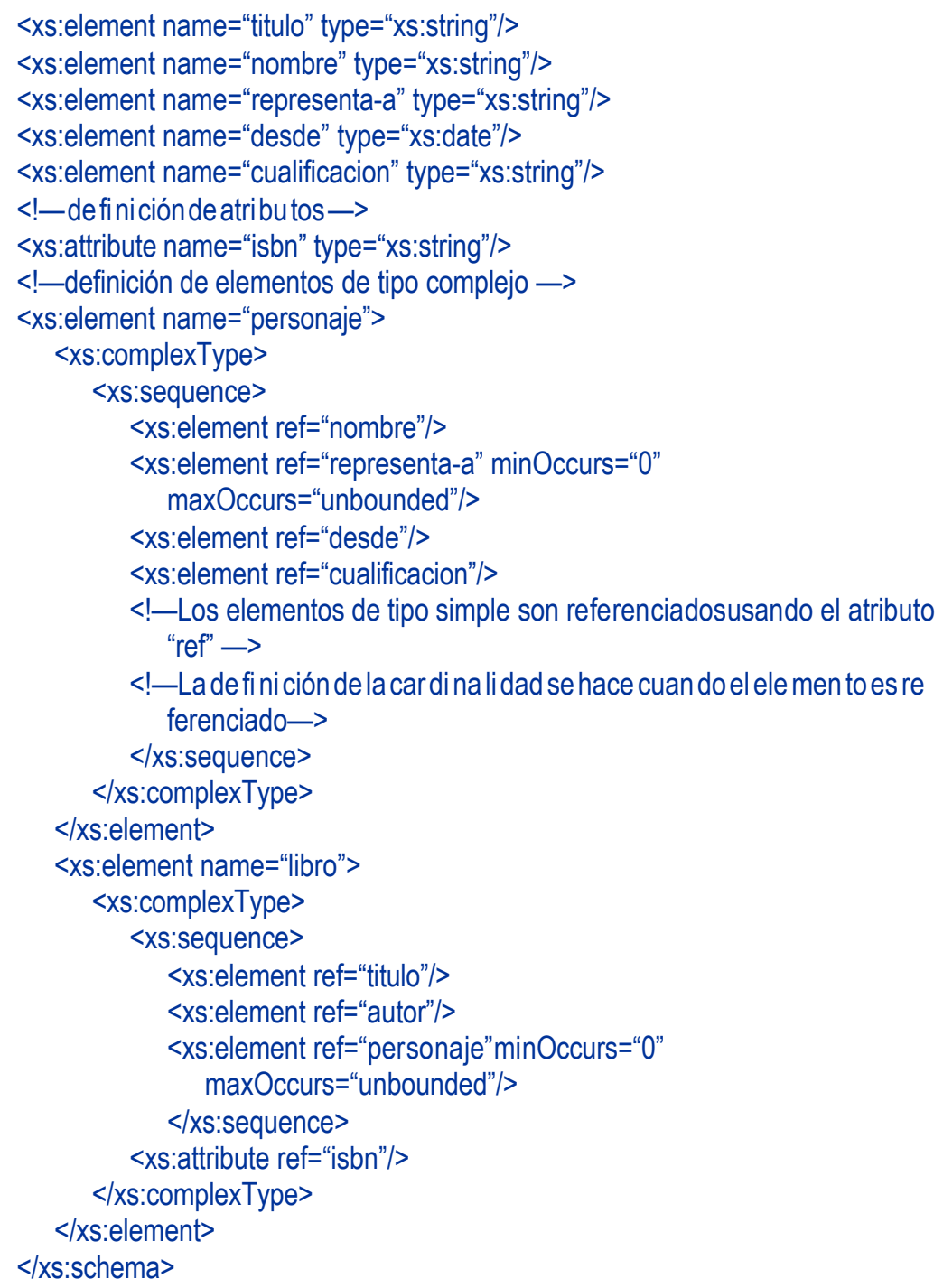

Usar una re ferencia hacia un elemen to o atribu to es el equivalen te de "clo nar" a un objeto. Elelemen to oatribu to es de finido primeroyluegopuede serduplica do enotro lugardela es truc tu radeldo cu men to porelme ca nis moderefe rencia.Los dos elementosoatributos sevuelven deesta formadosinstancias dela mis maclase.

Hemos establecido ya que podemos definir elementos y atributos conforme los vamosnecesitando(técnica matriushka), ocrearlosprime royre ferirlos después (tabla plana).Eldo cu men to dees que masXMLdesarrolladoporelcon sorcio W3C nos proporciona un tercer mecanismo usado para definir dataTypes (tipos de datos) ya sea simples o de tipo complejo, y utilizar esos tipos para definir atributos y elementos. 
Esto se lo gra dán do les un nom bre a los ele men tos simpleType y complexType y ubi cándolos fue ra dela de fini ción de ele men tos o atribu tos. Más aún, po de mos derivarun tipo de da tos data Type ha cia otro de fi nien do una res tric ción so brelos va lo res de este tipodedatos.

Por ejem plo, para de fi nirundataTypellamado "tipo-de-nombre"elcualestaráformadoporunacadenadehasta32caracteres, loestableceríamosasí:

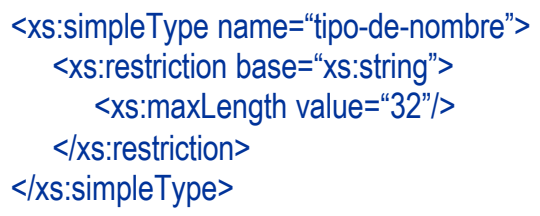

Elelemento simpleType guarda el nom bre del nuevo dataType. Elelemen to restriction ex pre sa el he cho de que eldataType se de riva del dataType "string" del es pa cio de nombres de W3C (namespace), pero se le aplica una restricción a sus posibles valores. El atributo maxLength, tambiéndenominado faceta en los do cumen tos del con sorcio, es pecificacuálesesarestricción, conunalongitudmáximade 32 caracteres.

Otra "face ta" pode rosaes el elemen to pattern (pa trón), el cual de fine una propo sición regularque debe sersatis fe cha. Por ejem plo, sinonosinte re sa de finirelele mentoisbnconsusguiones, podríamos de finirel dataTypeisbn como:

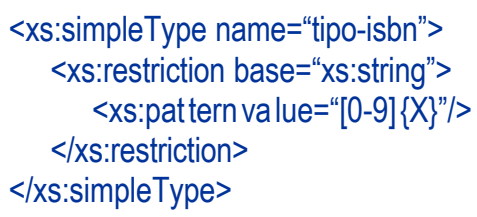

Es de cir,lo de fi ni mos como una ca dena de ca rac te res cuyo pa trón po sible sonlos núme ros 0-9 (ceroal nueve) yel“número"X(diez),única mente.Los guiones que dan por ende ex clui dos. Sinosin te re sa ra agregar el guión como po sible en el pa trón, simplemente agre ga mos el va lor del guión $\{-\}$ des pués del va lor del nú me ro X; esto es: "[0-9] $\{\mathrm{X}\}\{-\} "$

DefiniryutilizardataTypesconnombrees com parableade finirunaclaseyutilizarla para crear un ob je to. Un dataType es un ente abs trac to que pue de serutiliza do para de finirun ele men to oun atribu to. El dataType rea liza en ton ces con un ele men to o un atributolamismafunciónqueunaclaserealizaconunobjeto.

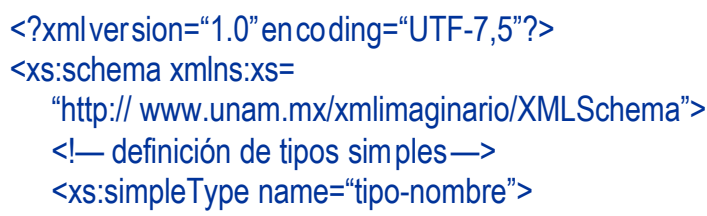




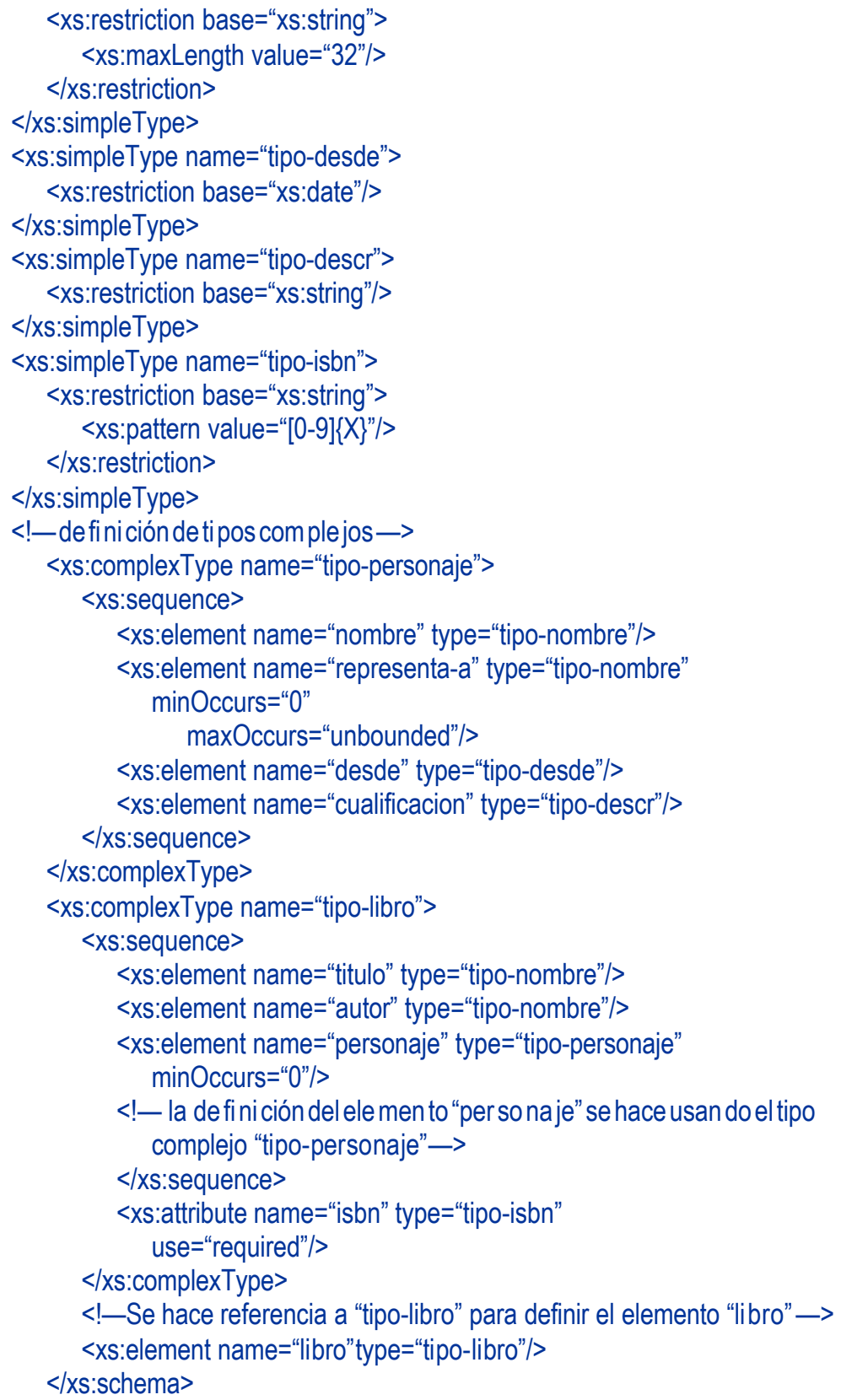




\section{Grupos, compositores y grupos derivados}

Los es que mas de acuerdo conelW3Ctambiénpermitenlade finición degrupos de elementosyatributos:

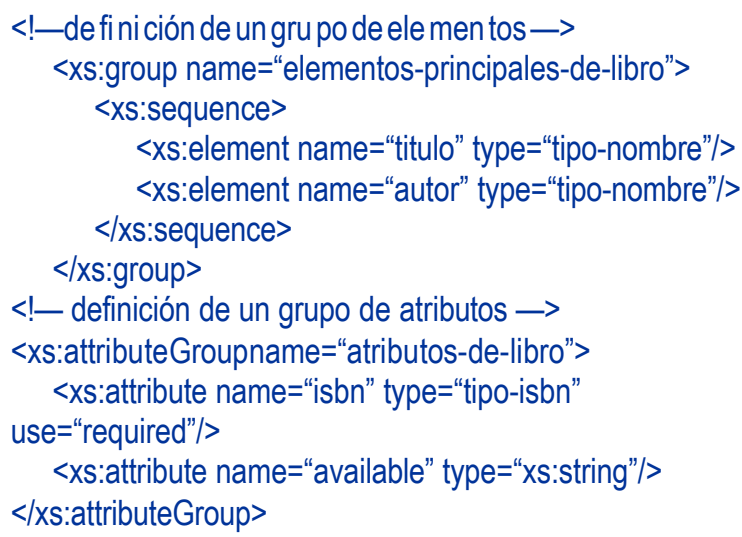

Estos grupos pueden ser usados en la definición de tipos complejos, como se muestra:

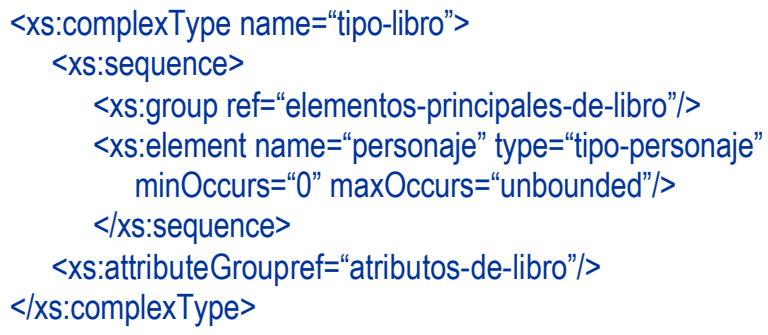

Es tos gru pos no son dataTypessino con tene do res de un conjun to de ele mentoso atributosquepuedenserusadosparadescribirtiposcomplejos.

\section{Compositores}

Hasta ahora hemos visto el compositor de secuencia xs:compositor que define gruposordenadosdeelemen tos opartículas, loscuales pueden serasuvezgruposde otroscompositores. ElXML del W3Csoportaademás doscompositoresadicionales que pue den combinar se para permi tirva rias mez clas. Cada uno de esos com po si to res puede te ner un mínimo y un máxi mo de ocu rren cias (minOccurs y maxOccurs) paradefinirsucardinalidad. Unboundedsignificasinlímite. 
Elcompositor xs:choice des cribela elecciónentrevariosposibles elementosogrupos de ellos. El siguien te grupo (los compo si to res pue den apa recerden tro de grupos tipos com plejos u otros com po si to res) acep ta bien un sim ple nom bre de ele men to o una secuenciadenombre-de-pila,unapellido-paternoounsegundo-apellidoopcional:

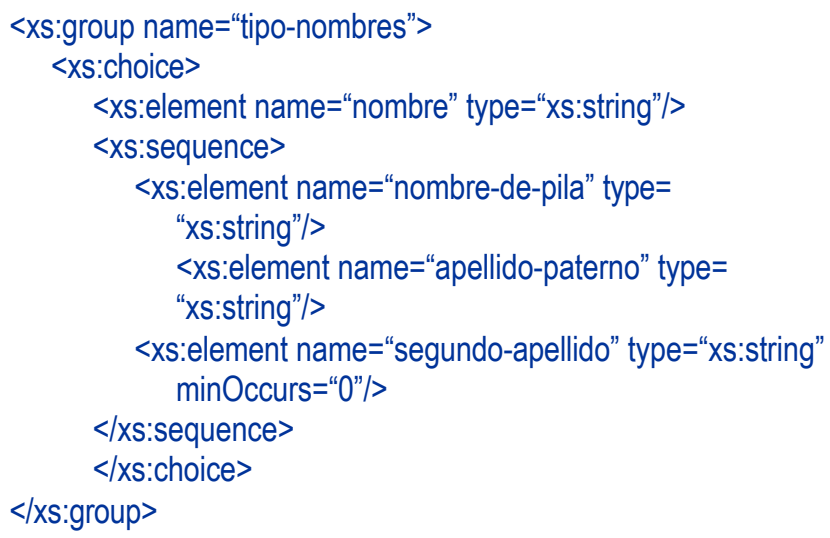

Elcompositorxs:allde fineunconjun to no-ordenado deelemen tos. La siguiente de finición de tipos com plejos permite a sus ele men tos con tenidos aparecerencuat quierorden:

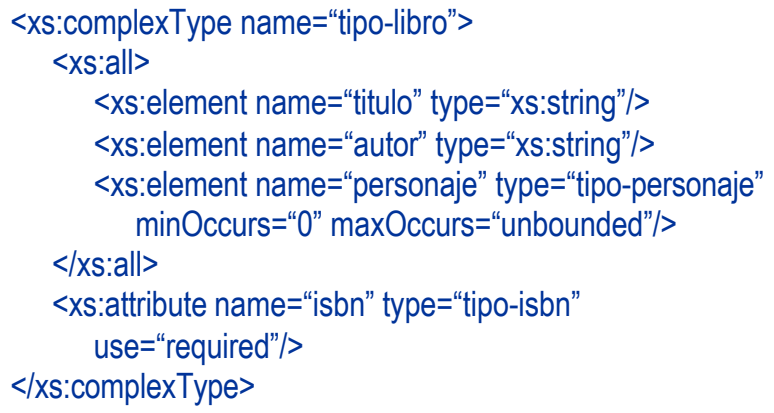

Paraevitarcombinaciones quepu die ran re sul tarambiguas odemasia do complejas para serre suel tas porlas he rra mien tas del es que maXML deW3C, deben agregarse unconjuntoderestriccionesaladeclaración xs:all.

Sólo pueden aparecer como un hijo por una vez al principio del paquete Sus hijos pueden ser sólo definiciones xs:element o referencias y no pueden tener una cardinalidad mayor que uno. 
Derivados de tipos simples

Los dataTypes sim ples pue den serde fini dos por de riva dos de otros dataTypes, bien predefinidos e identificados por el namespace del esquema del W3C o definidos en otroladoen elesquema.

Hemos visto ya ejemplos de tipos simples derivados por restricción (usando xs:restriction).Las varieda des diferentes de res tric cionesquepue denseraplicadas aun dataTypesellamanfacetas,comoyahemos establecido,y son particularmenteútiles para es ta ble cerla lon gitud de un ele men to, los po sibles valo res que pue de to mar, el númeromínimoymáximodeocurrencias,etcétera.

Exis tenotros dosméto dos de de riva ción que permi ten de finires pacios enblan co, lis tas sepa ra dasparasercon sultadas, uniones de dataTypes, etcétera.Lasiguientede fini ción usaxs:union paraex tendernues trotipo-isbnyqueaceptelosvalores TDB yNA:

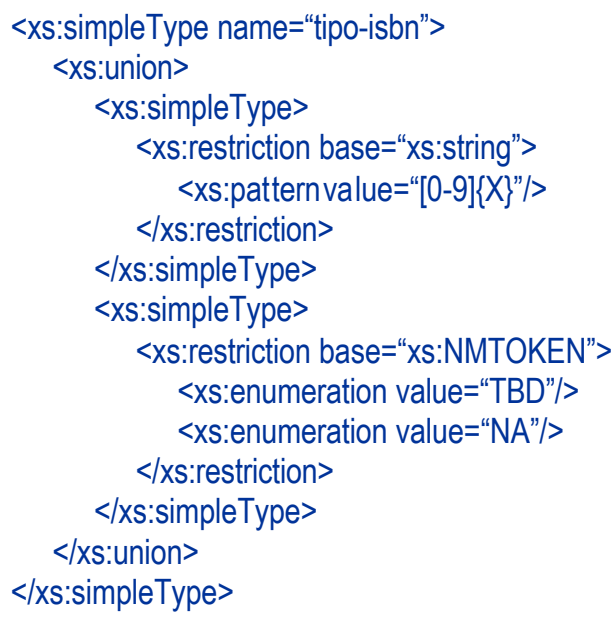

La "unión" ha sido aplicada a dos tipos distintos de valores para permi tir la existenciadedos conjun tos devalores; el nuevo dataTypeacep ta aho ralos valoresenumeradosTBD yNA.

Elsiguienteejemplo “tipo sisbn" usaxs:list para de fi nir una lis ta de va rios ISBN se para dos por un es pa cio en blanco. De riva tam bién un tipo "tipo-isbn10" usandoxs:restriction paraaceptarentre1 y10valores denúmerosdeISBN separadosporunespacio.

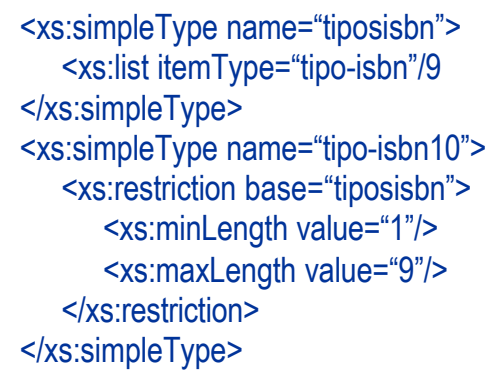


Mezclas en los contenidos

Larecomendacióndel W3C para esque masnos permite también mezclarpartes de documentos marcados con otras secciones sin marcar usando la restricción xs:complexType;obsérveseelsiguientetexto:

$<$ isbn libro="8420639028">

Obra dramáticade JoséZorrilla

Su título, Don Juan tenorio, lo dice todo!

$</ i s b n$ libro>

Usandolarestricciónxs:complexType:

$<x$ :element name="libro">

$<x$ s:complexType mixed="true">

$<x$ s:all $>$

$<x$ s:element name="titulo" type="xs:string" $/>$

$<x$ s:element name="autor" type="xs:string"|>

$</ x s: a l l>$

$<x$ :attribute name="isbn" type="xs:string"/>

$</ x \mathrm{x}:$ complexType $>$

$</ x$ s:element>

Locualvalidaráunelemento XMLcomoelsiguiente:

$<$ isbn libro="8420639028">

Divertida obra por <autor>José Zorrilla</autor>.

Su título(<titulo> Don Juan tenorio</titulo>) lo dice todo! $<$ <isbn libro>

La construcción xs:keyref es súmamante útil en este tipo de obras ya que permite ha cerligas en tre al gu nos ele men tos y otros. Por ejem plo, de sea mos aso ciara los dis tintoselementos "personajes" den trodeestaobra;recordemoslade finiciónes tablecidaenunprincipio:

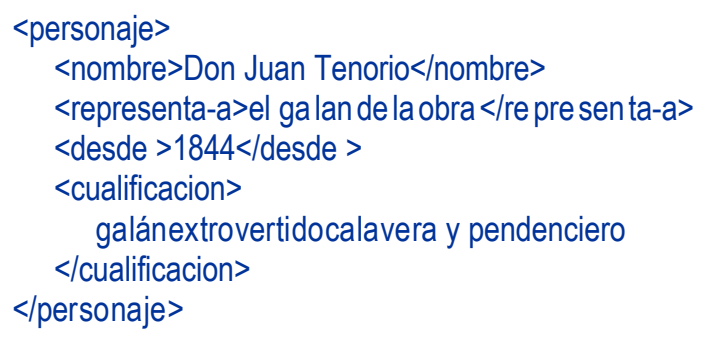


Y queremos indicar que hay una asociación entre este personaje y otro u otros den tro de la obra; por ejem plo, para que cuan do uno sea con sul ta do, se aso cie siemprealotro, el cualdebeserre ferencia do en el mismolibro:

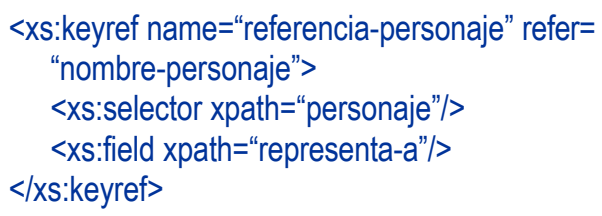

Nospresentaráasociacionesconotrospersonajesdelaobra;porejemplo:

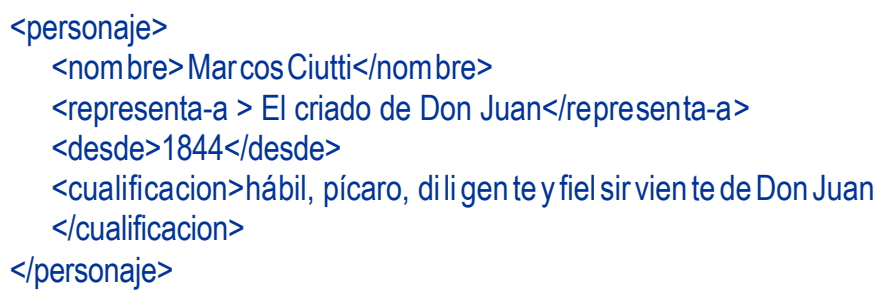

Existen todavía algunas otras definiciones para la completa construcción de un es que ma, pero no es el caso de este do cu men to con so lidarse como un tu to rial de esquemas, sinoelhaberilus tradolasconsiderablementemayoresposibilidadesqueun esquema permite lograr mucho más allá de la simple definición DTD de un documento.[W3Cschools, 2003]

\section{LENGUAJES PARA ESQUEMAS}

Por todo lo anteriormente expuesto sobre la estructura de esquemas, es claro que nospermiten es tableceruna enormecantidaddeca racterís ticas para el registro, elcontroly elcon tex to de un documen to dado, perola es truc tura pue dellegaracomplicarse tanto que la construcción de un esquema paso a paso parecería una enorme tarea, comoaquíhemos podidoobservar.Dalaimpresión de quedebeunomemorizarenor mescantidadesdeinstrucciones, símbolos, variables, estructuras, etcétera,locual a primera vistapare cete dio soyatemo rizan te.Peronoesasí.Enlaprácticalosesquemas no tiendenaserescritosporlaspersonassinoporlas máquinas.

Comohemospodidoobservar,la especifica ción 1.0 de XMLde finelos conceptos paraunacorrecta formabilidadyvalida ción. A tra vés deellaes muy fácilve rificarun do cumento cer tificandoque tengabuena formabilidad;noobs tante,lavalidación requiere de más trabajo y le permite al usuario definir restricciones más potentes para la estructura del documento. La validación XML requiere que el documento 
siga las restricciones es tablecidas enelDTD, el cual provee el equivalente de lo que seríaenuncon tex tolibreunasin taxis paraun tipodedocumen to [Eito,2001].

Enalgu nosusoslos siste mas pue den re que rirde finiciones de marca do másin formativas, orestric ciones más es tric tas o más flexibles enla es tructu radeldocu mento, o simplemente diferentes de las expresadas en definiciones DTDs según las define XML1.0. Hay tambiénuna ten denciaapermitirmarcados y res tric ciones quepuedan serespecificadosenunasintaxis totalmente XML, para que de esta forma las diversas herramientas electrónicas para los documentos XML pue dan ser usa das en las mismas especificaciones. Esto puedelograrsegraciasala creacióndelen guajes de programaciónparalacreacióneinterpretacióndeesquemasdemarcado.

De la información proveniente de la página del consorcio W3C para los requerimientos de un esquema [Malhotra, 1999] podemos diferir que el propósito del lenguaje de es que mas XMLes el de pro veer un in ven ta rio de las cons truc cio nes de marcado XML con las cuales se pue den es cribir es que mas. A su vez, el pro pó si to de un es que ma es de finirunaclasede do cu men tosXMLy usar esas cons truc cio nes para delimitary docu mentarel significa do, uso y rela cio nes de sus partescons ti tuyen tes: tiposde datos, elementosysuscontenidos, atributosy sus valores, en tida des ysuscontenidos y notaciones, valores implícitos como por ejemplo los de omisión (default), etcétera.Loses que mas documentan su propio significado, usoy función. Porello, el len guaje de es que masXML pue de serutiliza do para de finir, des cribiry ca ta logarvocabularios XMLparadiversasclases de documentos XML. Todo progra maXML puedeusarelformalis mo inheren tea un es que ma para ex presardelimi ta ciones devalor, estructuralesosintácticas,aplicablesaciertosdocumentos.

Algunos delos po sibles ambientes, ade más deldelas biblio tecas digitales, quepodrían verse beneficiados con el uso de la programación basada en estos esquemas puedenser:eledito rial, dondela dis tribucióndein formaciónpuedeinvolucrardocumen tos que guardan com plejas rela cio nes en tre sí (en es tas rela cio nes los es que mas estructurales describirían las relaciones entre encabezados, nuevos artículos, referenciascruzadas, fo togra fías, etcétera); elcomercioelectrónico;laadquisición masiva de datos; la transferencia de in forma ciónen tre ba ses de da tos; elin tercambio de metadatos,etcétera.

De acuerdo con el grupo de trabajo de esquemas del consorcio W3C, los principiosdediseñoparaunlenguajedeesquemassebasanenlassiguientescaracterísticas:

Ellenguajeparaesquemas XML debe:

* Ser más expresivo y detallado que los DTDs.

* Poder expresarse totalmente en XML.

* Ser autodescriptivo.

* Ser utilizable por una amplia variedad de programas que usen XML.

* Poder ser ampliamente utilizable en el Internet.

* Estar optimizado para interoperabilidad. 
Ser suficientemente simple para poder implementarse con recursos moderados.

* Estar coordinado con otras especificaciones W3C (ligas, apuntadores, etcétera).

En cuanto a sus requerimientos estructurales, el lenguaje para esquemas XML debepoderdefinirlosmecanismospara:

* Delimitar la estructura de los documentos (espacios, elementos, atributos) así como de los contenidos (tipos de datos, entidades, notaciones).

* Habilitar la herencia para definir elementos, atributos y tipos de datos.

* Habilitar documentación autocontenida dentro de otra.

* Habilitar descripciones y delimitadores específicos para un programa en especial.

* Contender con la integración de esquemas estructurales con tipos de datos primitivos.

* Definir las relaciones entre el esquema y los documentos XML.

* Definir las relaciones entre esquemas y DTDs.

De lo anterior po de mos con cluir que en los pró ximos años ha brá una ten den cia marcada para cons truiry desarrollarlenguajes deprogramación quepermitanlacrea ción y explotación de esquemas en una forma mucho más sencilla de la que hemos idoestableciendoaquíen este documento. No perece factiblequelas perso nas sedediquena es cribirlíneasylíneas con todas esas especificaciones y res tricciones. L o que sí es necesario es que se entiendan los conceptos básicos que permiten definir, restringir, correla cionar, et cétera, alas dife ren tes en tidades deundocumen to; sería imposible hacerlo de otra forma. Alguien tiene que establecer las características y estructurasdeundocumento.

Sin embargola gene ra ción en sídel có di go del es que ma será fa cili ta da a tra vés de lenguajes para esquemas. Algunas pantallas amigables al usuario nos permitirán trans mi tir de ma ne ra sen cilla la idea que nos he mos he cho del es que ma en múl tiples ins truccionesqueseránleídasporotros programasparainterpretaryprocesar.

Lo im portan te de todo esto, en suma, es apren der a crear la es truc tu ra men tal de ordenycon tex to queundocumen to conlleva, de acuerdoconla filosofía delos esque mas que, como hemos podido observar, permite contextualizar los documentos mucho más allá delo quehe mos es ta do acos tumbrados. Des demi pun to devis ta, el aprendera desarrollares que mas noes triba enla habili daddees cribircó digos, sinoenla habilidad deplan tear se undocu men to enla men te bajounagran es truc tu ra organiza tiva, y creo que eso es lo más rele van te del apren di za je delos es que mas, so bre todo para los biblio tecarios que nos hemos acostumbrado a disectar, catalogar y acomodar los múltiples ele mentos de una fi cha. Mepa re ce que el es que ma nos permi te aplicar las es truc tu ras deorden y sis te ma no tan solo a los ele men tos ca talográ fi cos, sinoal con te nido mis mo deldocumento.Deahísugranvalorcomoeducadoresdelamente. 


\section{CONCLUSIONES}

Comohapodidoobservarse, elmarcado XML, aun en un sim pleDTD, permi te establecerdefinicionesmuchomáscompletas deundocumen toquelasquepodíamos es tablecerenuna fichaca talográ ficaoundocumento HTML para des plie gue. Es claro el po ten cial que el mar ca do XML nos permi te de sa rrollaren el marca do y la re cuperacióndocumental,aunaniveldeunDTD.

Pero, como hapo didocom pro barse, las es truc tu ras plantea das porXML pue den irse so fis tican do másy más. La propues ta decrea ción de esque mas rela ciona dos con un tipo dado de do cu men to nos ofre ce un po ten cial aún mu cho ma yorde re gis tro y explotación de la in formación, sin per derla cali dad de re gis tro ca ta lo gráfico,como estamosacostumbrados, sinoextendiéndoloanivelesmásprofundosdelcontenido.

Los esquemas pue den via jar en la red jun to con los do cu men tos marca dos bajo su es truc tu ra. Esto quie re de cirque el nively calidad de nues tro marca do no está cons treñido a nuestro sistema de cómputo. Podemos entregar e intercambiar documentos cones tructuras muycomplejasypreci sas quevayanllevan doaniveles máspro fundos, profesionalesysatisfactoriosdealmacenamientoyrecuperacióndeinformación.

Comopuedededucirseesimportanteircreandoproyectosquedesarrollenesquemas específicospara dis tin tos tiposdedocumentosden trodelasbibliotecasmexicanas. Ello tendría un gran valor a futuro para las nacientes bibliotecas digitales de nues tro país, ya que po dría ofre cerse un es tán darmexica no y, sinem bargo, perfecta mente internacional, hecho por pro fe sio nales, y con el cual pue dan esas biblio te cas empezara "marcar"sus docu men tos de acuerdoconlasugerencia de cadaes quema. Enprimerlugar, po dría re solverse de unavezla dis cusión teó rica so bre siusar DTDs oesquemas; o DTDsy es que mas. Con esere sul ta do, y de pen dien do del tipo dema terial, cada biblioteca podrá entonces alojar documentos en bibliotecas digitales de tipo "no vela", "obra de tea tro", "libro de tex to", etcé te ra. A la lar ga, el pro yec to podría con ti nuarse para ob te nerun con jun to de to dos los forma tos "típicos" de documentos:libros, revistas, mapas, discos,películas, etcétera, comoalgunavez MARClo hizo, pero con divisionesespecíficaspara cada variedad de documento en cada formato, y con alcances para cada par te del con te ni do y no sólo para los da tos de re ferencia. Esto aseguraría un buen niveldemarca do, la estan da riza ción en tre diversas bibliotecas digitales mexicanas que se vayan creando y, lo más importante, una esplén di da y po de ro sa re cu pe ra cióny ex plo ta ción por parte delos usua rios, etcéte ra. Con ello podrían sentarse las bases para un modelo de desarrollo de esquemas que pu die se ser lle va do a nivel de re co men da cio nes para el me dio me xi ca no y talvez ser imitadoenotrospaíseslatinoamericanos.

Sinoiniciamosunproyectodeeste corte, enunfuturocercanolas bibliotecas empezarán a hacer sus marcados XML con for me al di se ño de cada una de ellas, y como no exis teningúnestán dar, habrámuy diferentes especifica cionesycalidadesdemarcado, in clu so al gu nas que nada ten gan que ver con este sec tor. Esto no se ría bue no paraesas colecciones digitales, pues cuando se deseara adoptar algún estándar preestablecido 
quizásería tardeypodrían exis tirmu chos documen tos yain troducidosenalgunacolec ción dada, locualdificul ta ría la conver sión a nue voses tán da res yobs ta culiza ría la estandarizacióndemarcadosycalidadeshomogéneas paralascolecciones electrónicas. Peor aún se ría tenerque es pe rareimi tares tán da resin terna cio nales, que sibien pue den es tarbien he chos nos siguen dejandoala som bra de esos de sa rrollos he chos enotrospaíses, nodeltodoacordesconnuestrarealidadnacional.

Finalmente,lasconclusionespuedenresumirsedeacuerdoconlosiguiente:

Los lenguajes de marcado realmente no son nuevos; tienen más de cuatro décadas y lo que observamos hoy son sus versiones más sofisticadas.

* Los metalenguajes usados hoy en día en el mundo electrónico-documental tienen diversascaracterísticas, ventajas y desventajas dependiendo del contexto de su creación y su momento histórico.

* La ten den cia hoy en día es que los len gua jes de mar ca do ayu den a con sig nar no tan solo los as pec tos ca talo grá fi cos de una obra, sino también los rela cionados con su contenido.

* XML se cons titu ye comola versión más com pletay fun cio nal deloslengua jes de marcado hoy en día.

* Los esquemaspermitenla de finiciónde estruc turasy relaciones mu chomás completas que las de un DTD.

* El establecimiento de lenguajes de creación de esquemas facilitará la parte operativa y mecánica del desarrollo de esquemas.

* Es importante ir creando definiciones, DTD o esquemas, de las principales clases dedocumentosenelmediobiblio te cariomexicano, paragarantizarsu calidad y profundidad.

* Esimportante en ten derla filoso fía decreación de esque mas comoun mecanis mo de ejercicio mental de organización y sistematización de la información.

\section{REFERENCIAS BIBLIOGRÁFICAS ${ }^{1}$}

A general Introduction to the MARC Format. Disponible en: http://www.tlcdelivers.com/tlc/crs/Gen0009.htm

Campbell, Ch. 2003. "XMLSchema”. En: Sig mond Re cord, Vol. 32, No 2, Junio 2003. pp. 96-101.

Eito, R. 2001. Programación con XML. Madrid: Anaya. ISBN: 84-4151186-1. pp. 487-540.

* Exten sibleMarkupLanguage (XML) 1.0 (2nd. ed.), W3C Recommendations. Octubre 6, 2002. Disponible en: http://www.w3c.org/TR/1998/NOTE-XML-data-0105/

Gu tie rrez, A y Martínez, R. 2001.XML a tra vés de ejem plos. Ma drid:RAMA. ISBN: 84-7897-455-5.

1 Los URL'S mar ca dos con un* co rres pon den a un si tio ofi cial o con ni vel de es tán dar in ter na cio nal. 
Free Dic tio nary of com pu ting. 2000. En tra da por: "Backus-NaurForm". Disponible en:

http://burks.bton.ac.uk/burks/foldoc/

* Iflanet. What is MARC. Disponible en: http://www.ifla.org/VI/3/p1996-1/unimarc.htm

* Library of Congress. MARC Standards. 2003. Disponible en: http://www.loc.gov/marc

* Library of Congress. MARC 21 XML Schema. 2003. Disponible en: http://www.loc.gov/standards/marcxml

Mo rrison, M. 2002. Teach Your selfXMLin 24 hours. In dia na: Sams.ISBN: 0-672-32213-7. Parte II, hora 5, “Using XML Schema”, pp. 69-95.

MSDN on line. 2000. XML Developing Center Disponible en: http://msdn.microsoft.com/library/default.asp?url=/nhp/Default. asp?contentid $=28000438$

* SGML ISO 8879. 1986. Disponible en: http://www.iso.org/iso/en/CatalogueDetailPage.CatalogueDetail? CSNUMBER $=16387$

Should it be an element or a type ? Disponible en: http://www.xfront.com/ElementVersus'Type.html

Teas da le, G. 1995. L'Hyper tex te: his to ri que et appli ca tions en bi blio théconomie. Université de Montréal. Disponible en: http://www.infotheque.info/ressource/942.html

Timeline of XML Events. Disponible en: http://www.ukoln.ac.uk/web-focus/events/workshops/webmaster -2002/materials/savory/slides/img18.html

* XHTML1.0: TheeXtensibleHypertextMarkupLanguage. Disponibleen: http://www.w3.org/TR/xhtml1/\#xhtml

The XML Cover Pages - Home page. Disponible en: http://oasis-open.org/cover/

[Malhotra, A. 1999]. XML Schema Requirements. Disponible en: http://www.w3.org/TR/NOTE-xml-schema-req

Van der Vlist, E. 2001. Using W3C XML Schema. Disponible en: http://www.xml.com/pub/a/2000/11/29/schemas/part1.html

Thompson, H. 1997. Why I demand "Schemata". Disponible en: http://www.ltg.ed.ac.uk/ ht/sgml97.html

* W3C Schools. 2003. XML Tutorial. Disponible en: http://www.w3schools.com/xml/default.asp

Young, M.J. 2002. XML Step by Step. $2^{\mathrm{a}}$ ed. Redmond: Microsoft Press. ISBN: 0-7356-1465-2. Capítulo 7: "Creating valid XML documents using XML Schemas”. pp. 163-192.

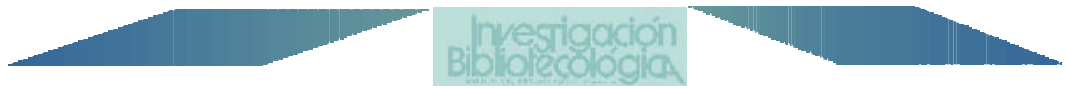

